\title{
Complex Chromatin Motions for DNA Repair
}

\author{
Judith Miné-Hattab ${ }^{1,2 *}$ and Irene Chiolo ${ }^{3 *}$ \\ ${ }^{1}$ UMR 3664, CNRS, Institut Curie, PSL Research University, Paris, France, ${ }^{2}$ UMR 3664, CNRS, Institut Curie, Sorbonne \\ Université, Paris, France, ${ }^{3}$ Molecular and Computational Biology Department, University of Southern California, Los Angeles, \\ CA, United States
}

OPEN ACCESS

Edited by: Karim Mekhail,

University of Toronto, Canada

Reviewed by:

Jean-Yves Masson,

Laval University, Canada

Jean Gautier,

Columbia University Irving Medical

Center, United States

*Correspondence:

Judith Miné-Hattab

judith.mine@curie.fr

Irene Chiolo

chiolo@usc.edu

Specialty section:

This article was submitted to Epigenomics and Epigenetics,

a section of the journal

Frontiers in Genetics

Received: 25 March 2020

Accepted: 06 July 2020

Published: 27 August 2020

Citation:

Miné-Hattab J and Chiolo I (2020)

Complex Chromatin Motions for DNA

Repair. Front. Genet. 11:800.

doi: $10.3389 /$ fgene.2020.00800
A number of studies across different model systems revealed that chromatin undergoes significant changes in dynamics in response to DNA damage. These include local motion changes at damage sites, increased nuclear exploration of both damaged and undamaged loci, and directed motions to new nuclear locations associated with certain repair pathways. These studies also revealed the need for new analytical methods to identify directed motions in a context of mixed trajectories, and the importance of investigating nuclear dynamics over different time scales to identify diffusion regimes. Here we provide an overview of the current understanding of this field, including imaging and analytical methods developed to investigate nuclear dynamics in different contexts. These dynamics are essential for genome integrity. Identifying the molecular mechanisms responsible for these movements is key to understanding how their misregulation contributes to cancer and other genome instability disorders.

Keywords: chromatin motions, double-strand break repair, homologous recombination, mean square displacement, directed motion, multi-scale motion

\section{INTRODUCTION: CHROMATIN EXPLORES A LARGER NUCLEAR VOLUME IN RESPONSE TO DNA DAMAGE}

A significant number of studies in the past decade have identified essential roles for nuclear dynamics in DNA repair, particularly during homologous recombination (HR) repair of doublestrand breaks (DSBs) (Figures 1A-D). First, a larger nuclear volume explored by repair sites is typically detected during inter-homolog recombination (Figure 1A) (Miné-Hattab and Rothstein, 2012; Neumann et al., 2012; Cho et al., 2014; Miné-Hattab et al., 2017) (reviewed in Dion and Gasser, 2013; Mine-Hattab and Rothstein, 2013). This change in chromatin mobility in response to DNA damage likely reflects the exploration of the nuclear space during "homology search" (Kalocsay et al., 2009; Dion et al., 2012; Miné-Hattab and Rothstein, 2012; Neumann et al., 2012; Agmon et al., 2013; Cho et al., 2014; Saad et al., 2014; Herbert et al., 2017; MinéHattab et al., 2017), i.e., the process where a resected DSB covered by a Rad51 nucleoprotein filament scans the genome in search of a homologous donor. Second, undamaged chromatin also becomes more dynamic during DSB repair, albeit to a lesser extent than repair sites (Figure 1B) (Chiolo et al., 2011; Krawczyk et al., 2012; Miné-Hattab and Rothstein, 2012; Seeber et al., 2013; Lottersberger et al., 2015; Strecker et al., 2016; Herbert et al., 2017; Lawrimore et al., 2017; Miné-Hattab et al., 2017; Caridi et al., 2018a; Smith et al., 2019; Zada et al., 2019). The significance of the genome-wide increase in nuclear exploration is still under debate, but this response might increase the frequency of DNA contacts to facilitate homology search (Gehen et al., 2011; Neumann et al., 2012; Mine-Hattab and Rothstein, 2013; Amitai and Holcman, 2018), or reflect chromatin relaxation to promote access for repair (Kruhlak et al., 2006; Ziv et al., 2006; 
Seeber et al., 2013; Delabaere and Chiolo, 2016). Third, repair sites undergoing HR aggregate into larger units, or "clusters" (Figure 1C) (Lisby et al., 2003; Aten et al., 2004; Kruhlak et al., 2006; Chiolo et al., 2011, 2013; Krawczyk et al., 2012; Neumaier et al., 2012; Cho et al., 2014; Caron et al., 2015; Aymard et al., 2017; Caridi et al., 2018a; Schrank et al., 2018; Oshidari et al., 2019a; Waterman et al., 2019) (reviewed in Chiolo et al., 2013; Guénolé and Legube, 2017; Schrank and Gautier, 2019), likely to facilitate DSB signaling and resection, e.g., by increasing the local concentration of checkpoint and repair proteins (Chiolo et al., 2013; Schrank et al., 2018; Kilic et al., 2019; Oshidari et al., 2019a; Schrank and Gautier, 2019). This response also occurs in G1 in human cells (Aten et al., 2004; Aymard et al., 2017), where HR cannot be completed with the sister chromatid, and clustering might temporarily sequester breaks that will be repaired in $S$ phase (Aymard et al., 2017). Clustering can also be deleterious, as increasing the proximity of DSBs on different chromosomes promotes chromosomal translocations (Agmon et al., 2013; Roukos et al., 2013; Lee et al., 2016; Cohen et al., 2018). Fourth, DSBs relocalize to specific subnuclear compartments when the lesion occurs in DNA regions that are difficult to repair. Specifically, DSBs in pericentromeric heterochromatin relocalize to the nuclear periphery in Drosophila cells (Figure 1D) (Chiolo et al., 2011; Ryu et al., 2015, 2016; Janssen et al., 2016, 2019; Caridi et al., 2018a), and to the periphery of heterochromatin "domains" (or "chromocenters") in mouse cells (Jakob et al., 2011; Chiolo et al., 2013; Tsouroula et al., 2016; Caridi et al., 2018a). rDNA sequences move to the nuclear periphery in budding yeast (Torres-Rosell et al., 2007; Horigome et al., 2019) and to nucleolar caps in mammalian cells (Harding et al., 2015; van Sluis and McStay, 2015; Korsholm et al., 2019; Marnef et al., 2019). Relocalization of repair sites to the nuclear periphery is also a response to damaged CAG repeats in budding yeast ( $\mathrm{Su}$ et al., 2015; Aguilera et al., 2020; Whalen et al., 2020), collapsed replication forks in yeast and mammalian cells (Nagai et al., 2008; Su et al., 2015; Lamm et al., 2018; Aguilera et al., 2020; Whalen et al., 2020), and damaged telomeric or subtelomeric sequences in yeast (Therizols et al., 2006; Khadaroo et al., 2009; Cho et al., 2014; Chung et al., 2015; Churikov et al., 2016; Oshidari et al., 2018; Aguilera et al., 2020). Similar relocalization occurs as a result of persistent/unrepairable DSBs (Nagai et al., 2008; Kalocsay et al., 2009; Oza et al., 2009; Horigome et al., 2014, 2016; Swartz et al., 2014; Marcomini et al., 2018). In these contexts, relocalization appears to prevent aberrant recombination with ectopic repeated sequences (Torres-Rosell et al., 2007; Chiolo et al., 2011; Ryu et al., 2015, 2016; Su et al., 2015; Caridi et al., 2018a; Dialynas et al., 2019; Aguilera et al., 2020) and/or promote alternative repair mechanisms (Therizols et al., 2006; Nagai et al., 2008; Khadaroo et al., 2009; Oza et al., 2009; Horigome et al., 2014, 2016; Churikov et al., 2016; Aguilera et al., 2020) (reviewed in Amaral et al., 2017; Caridi et al., 2017, 2019; Rawal et al., 2019). Further dynamics have been associated with other repair pathways. For example, deprotected telomeres are mobilized in mouse cells to promote non-homologous endjoining (NHEJ) (Dimitrova et al., 2008; Lottersberger et al., 2015). Additionally, a few chromosome territories reposition in response to damage in human fibroblasts, perhaps reflecting large-scale changes in chromatin organization (Mehta et al., 2010; Kulashreshtha et al., 2016). Several molecular mechanisms governing chromatin dynamics in response to DSBs have been identified, and specialized pathways appear to participate in different contexts [reviewed in Amaral et al. (2017); Caridi et al. (2017); Zimmer (2018); Oshidari et al. (2019b)]. Together, these studies revealed important roles for nuclear dynamics in DSB repair, particularly for homology search and for isolating repeated DNA sequences at high risk for aberrant recombination, enabling "safe" repair or alternative rescue pathways.

\section{METHODS TO CHARACTERIZE NUCLEAR DYNAMICS DURING DSB REPAIR}

Several techniques have been applied to study the nuclear dynamics of DSBs in different organisms, with "gold standard" approaches relying on damage induction with endonucleases or ionizing radiation (IR), and on monitoring repair sites with lacO/tetO arrays and fluorescent-tagged $\mathrm{HR}$ repair components (Figures 1E-G).

A widely used approach relies on the induction of sitespecific DSBs with an endonuclease (e.g., I-SceI, or HO), which recognizes a target sequence proximal to tetO or lacO arrays (Figure 1E). The position of the damage site is monitored using fluorescent-tagged TetR or LacI proteins that bind to the arrays, resulting in bright nuclear spots (or foci) (Robinett et al., 1996). Given that endonucleases can digest both sister chromatids, the sister is mostly unavailable as a template for repair, promoting inter-homolog exchanges (Miné-Hattab and Rothstein, 2012). In yeast, this approach enabled the study of nuclear dynamics associated with inter-homologous recombination (Miné-Hattab and Rothstein, 2012; Neumann et al., 2012; Miné-Hattab et al., 2017), unrepairable DSBs (Nagai et al., 2008; Kalocsay et al., 2009; Dion et al., 2012; Horigome et al., 2014, 2016; Saad et al., 2014; Herbert et al., 2017), sub-telomeric breaks (Khadaroo et al., 2009; Chung et al., 2015; Churikov et al., 2016; Oshidari et al., 2019b), and rDNA lesions (Torres-Rosell et al., 2005). Similar systems have been used to characterize DSB clustering in budding yeast and human cells (Lisby et al., 2003; Roukos et al., 2013; Waterman et al., 2019). A variant of this approach employs a lacO array close to the cut site and a tet $O$ array on a different chromosome, enabling the simultaneous tracking of both damaged and undamaged loci (Miné-Hattab and Rothstein, 2012; Miné-Hattab et al., 2017). These studies and others (Seeber et al., 2013; Herbert et al., 2017) revealed that not only damaged sites, but also undamaged chromatin explores a larger nuclear volume in response to DSB formation. An alternative system employed sequence-based tethering of oligomerizing fluorescent proteins that spread along the DNA, to study the dynamics of resected DNA (Saad et al., 2014). Here, resection results in loss of DNA-associated proteins and reduced signal at repair sites, and correlates with a local reduction in focus dynamics (Saad et al., 2014). These approaches are very powerful, but also limited to the site targeted by the endonuclease. Given that repair responses and relocalization pathways are affected by 
A Increased nuclear exploration
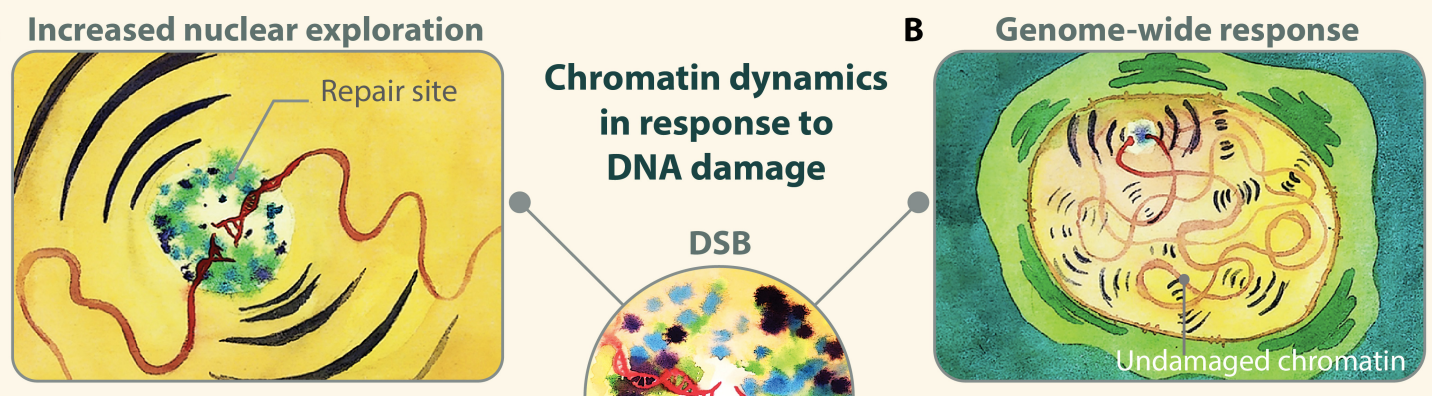

C Repair site clustering
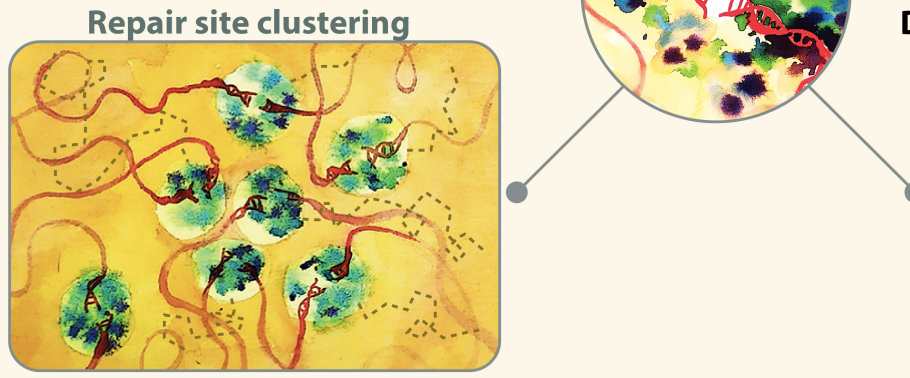

D

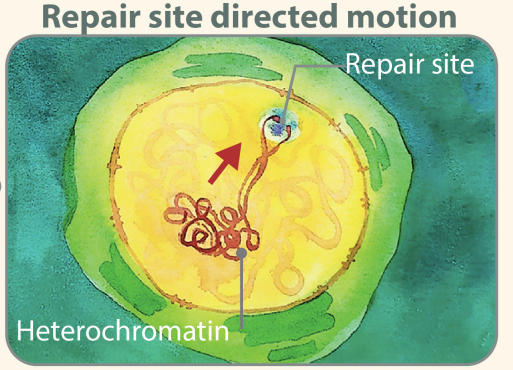

DNA damage induction approaches
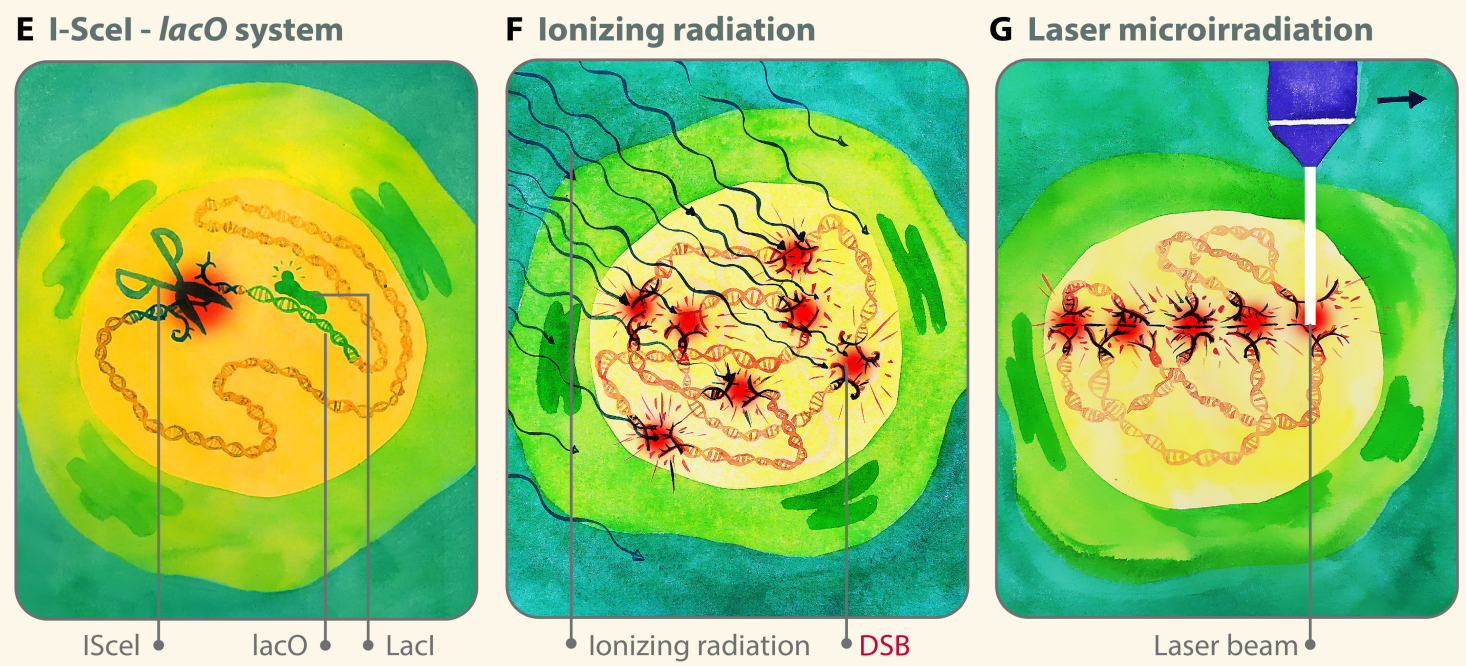

FIGURE 1 | Examples of damage-induced changes in chromatin dynamics. (A) A damaged chromatin site explores a larger nuclear volume during HR repair of DSBs. (B) Larger nuclear exploration is also observed for undamaged chromatin sites, indicating that the change in chromatin mobility is a genome-wide response. (C) Multiple DSB repair sites cluster together. (D) Damaged heterochromatic sites relocalize to the nuclear periphery with directed motions in Drosophila cells. (E,F) Three main approaches to study nuclear dynamics in response to DSBs rely on: (E) enzymatic digestion to induce damage and lacO or tetO arrays to follow damage sites; (F) IR to induce damage and fluorescent tagging of repair proteins to detect repair foci; (G) laser, alpha particles, or heavy ions, to induce damage along linear tracks where repair protein recruitment is detectable by live imaging or immunofluorescence. Illustration by Olga Markova.

chromatin state (Delabaere and Chiolo, 2016; Hauer and Gasser, 2017), nuclear positioning (Lemaitre et al., 2014), and the phase separated nature of nuclear subdomains (Altmeyer et al., 2015; Kilic et al., 2019; Lenzken et al., 2019; Oshidari et al., 2019a; Pessina et al., 2019) (reviewed in Clouaire and Legube, 2019; Rawal et al., 2019), the endonuclease-based approach would need to be applied to a large number of sites to provide a comprehensive understanding of the mechanisms responsible for these dynamics in different contexts.

Site-specific endonucleases have also been used to induce DSBs in highly repeated DNA sequences. For example, studies using Cas9 targeting heterochromatic satellites (Tsouroula et al., 
2016) or PpoI or Cas9 recognizing rDNA sequences (Harding et al., 2015; van Sluis and McStay, 2015; Korsholm et al., 2019; Marnef et al., 2019) revealed the dynamics of these sites in mammalian cells. It is important to consider that Cas9 affects the processing of repair intermediates (Richardson et al., 2016, 2018; Brinkman et al., 2018), potentially affecting outcomes and dynamics of repair.

Another commonly used approach relies on inducing damage with ionizing radiation (IR), and detecting repair sites using fluorescent-tagged HR components (Figure 1F). This has been an invaluable approach to characterize focus mobility relative to other nuclear structures, such as the heterochromatin domain, the nuclear periphery, or other repair foci (Kruhlak et al., 2006; Falk et al., 2007; Miné-Hattab and Rothstein, 2012; Chiolo et al., 2013; Lottersberger et al., 2015; Ryu et al., 2015, 2016; Caridi et al., 2018a,b; Schrank et al., 2018; Dialynas et al., 2019; Oshidari et al., 2019a; See et al., 2020). These studies established, for example, that heterochromatic DSBs move to the nuclear periphery to continue repair in Drosophila cells (Chiolo et al., 2011, 2013; Ryu et al., 2015, 2016; Caridi et al., 2018a). A major advantage of inducing DSBs with IR, relative to using chemical treatments or enzymatic digestion, is that IR-induced DSBs form within a very tight time window. This enables synchronous responses, and an easier characterization of focus dynamics and kinetics at the population level, including in animal tissues (see for example: Lisby et al., 2004; Delabaere et al., 2017; Caridi et al., 2018b; See et al., 2020). Further, IR treatments are well suited to damaging chromatin that is difficult to access with enzymatic digestion, such as heterochromatin (Goodarzi et al., 2008; Iacovoni et al., 2010; Chiolo et al., 2011; Ryu et al., 2015, 2016; Caridi et al., 2018a,b). A potential limitation of this approach is that tracking several sites in the nuclei requires frequent time points to minimize ambiguous tracks, which increases the potential for photobleaching and phototoxicity effects in long kinetics (Caridi et al., 2018b; See et al., 2020).

Alternative approaches employed lasers, alpha-particles, or heavy ions to induce damage along linear tracks in the nucleus of mammalian cells (Figure 1G), and repositioning of damage sites is monitored relative to these tracks and specific nuclear compartments (Aten et al., 2004; Chiolo et al., 2011; Jakob et al., 2011; Reynolds et al., 2013). These approaches revealed, for example, that repair foci cluster over time (Aten et al., 2004), and that damage in pericentric heterochromatin results in relocalization of repair sites to outside the chromocenters in mouse cells (Jakob et al., 2011). Using laser beams mounted on a microscope is also an effective method to investigate early damage responses (Bekker-Jensen et al., 2006). However, the high energy associated with some of these damage sources might also impair the chromatin state and repair outcomes (Singleton et al., 2002; Lukas et al., 2005; Reynolds et al., 2013; Kong et al., 2018), and even directly affect relocalization mechanisms (Chiolo et al., 2011).

Thus, several approaches have been developed to characterize the dynamics of repair foci. The preferred method depends on the type of question and the features of the motion under investigation.

\section{MSD ANALYSIS}

A traditional approach to characterize the dynamics of damaged DNA is the mean-square displacement (MSD) analysis of the positional data of repair sites (reviewed in Spichal and Fabre, 2017; Caridi et al., 2018b). The MSD curve represents the amount of space a locus explores in the nucleus, and its shape has been used to describe the nature of the movement (Michalet and Berglund, 2012; Oswald et al., 2014; Spichal and Fabre, 2017; Caridi et al., 2018b; Figure 2). The time-averaged MSD of a single trajectory is calculated using the following equation:

$$
\begin{aligned}
\operatorname{MSD}(n \cdot \Delta t)=\frac{1}{N-n} \sum_{i=1}^{N-n}\left[\left(x_{i+n}-x_{i}\right)^{2}\right. & \\
& \left.+\left(y_{i+n}-y_{i}\right)^{2}+\left(z_{i+n}-z_{i}\right)^{2}\right]
\end{aligned}
$$

where $N$ is the number of points in the trajectory, $(x, y, z)$ the coordinates of the locus in 3-dimensions, and $\Delta t$ the time interval of the acquisition. MSDs are typically calculated for several trajectories across distinct nuclei, and averaged to extract a time-ensemble-averaged MSD. The data are then fitted to a curve to characterize the type of diffusion. In the following sections, we present different models used in the literature to fit averaged MSD curves.

\section{Brownian Motion}

When a molecule freely diffuses, its MSD curve is linear at increased time intervals and its motion is called Brownian (Figure 2A). In this case, the MSD follows:

$$
\operatorname{MSD}(\Delta t)=2 d D \Delta t
$$

where $d$ is the dimension of the movement, $D$ is the diffusion coefficient of the locus, and $\Delta t$ is the time interval. The term $2 \mathrm{dD} \Delta t$ is the theoretical MSD for Brownian motion in the absence of any experimental noise. However, when measuring the position of a molecule in living cells, the experimental location accuracy can strongly affect the experimental MSD. The error in location for molecules detected by live imaging can be divided into two components (Supplementary Table S1):

- Error in the determination of the position due to convolution of the sample with the point spread function (PSF). This depends on imaging conditions and microscope features (e.g., the numerical aperture of the objective, the number of photons collected by the camera, and the wavelength of light). This error is higher at short acquisition times since the number of photons collected is small.

- Error due to the movement of the spot during the acquisition. This error, referred as "motion blur" is higher at longer exposure times.

Experimental MSD curves for Brownian motion, taking into account the location errors, can be fitted by Michalet (2010):

$$
\operatorname{MSD}(\Delta t)=2 d D \Delta t+\sigma_{0}^{2}\left(1+\frac{D t_{\operatorname{Exp}}}{s_{0}^{2}}\right)-\frac{4}{3} t_{\text {Exp }}
$$


A

Establishing how repair sites move in 3D is crucial to better understanding repair mechanisms that rely on nuclear dynamics. To quantify these movements, a classical method consists of calculating the mean square displacement (MSD), i.e, the mean squared distance covered as a function of the elapsed time.

\section{Brownian}
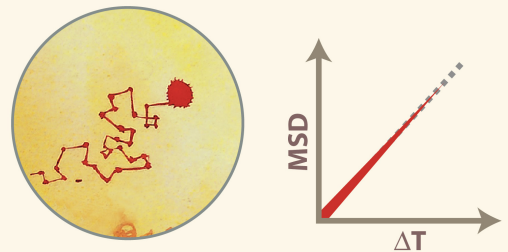

A particle with Brownian motion diffuses freely and explores all the available space with equal probability.

Anomalous
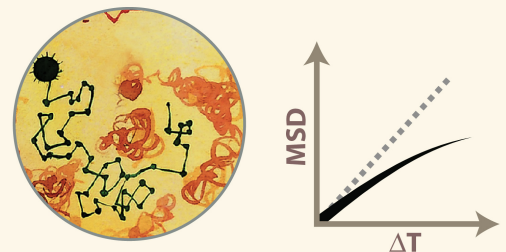

A particle with anomalous motion is constrained but, it can diffuse without boundary and reachs further targets if given enough time.

\section{Confined}
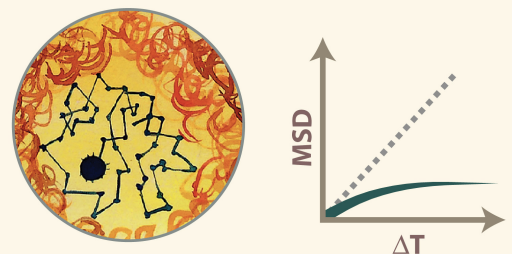

A confined particle remains inside a sub-volume from which it cannot escape.

Directed
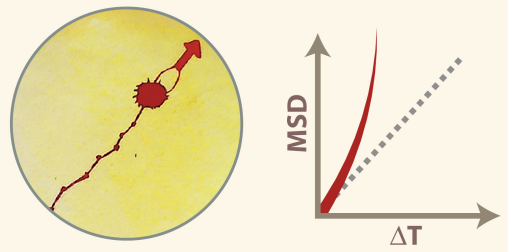

A particle with directed motion moves to a specific target.

\section{B}

The motion is often complex in living cells; two examples are shown here.

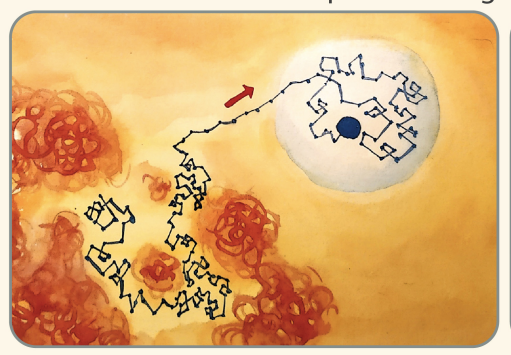

Mixed trajectory alternating anomalous, directed and confined motions.

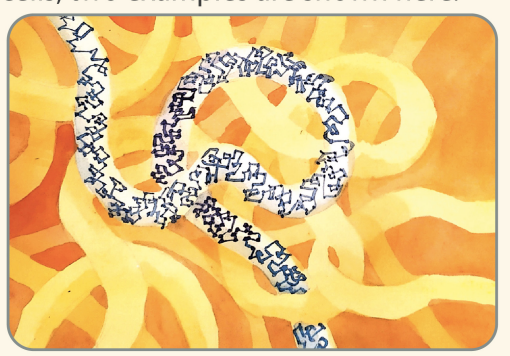

Multi-scale motion formed by the sum of two types of motions occuring simulatenously at different scales.

FIGURE 2 | MSD curves identify different types of motions. (A) Illustration of Brownian, anomalous, confined and directed motions, with corresponding MSD curves. (B) Examples of complex motion. Left: a mixed trajectory alternating anomalous, directed, and confined motion. Right: a motion of a site characterized by a diffusion coefficient Amicro, in a region that itself diffuses with a diffusion coefficient Amacro. Inspired by De Gennes's reptation model (Gennes, 1982). Illustration by Olga Markova. 
where $\sigma_{0}^{2}$ is the localization accuracy of an immobile particle; $s_{0}^{2}$ is the variance of the PSF;

$t_{\text {Exp }}$ is the exposure time of the camera.

\section{Sub-diffusive Motion}

In living cells, DNA motion is often slower than Brownian and is called "sub-diffusive" (Barkai et al., 2012). This is due to the existence of constraints that limit chromatin movement, including the polymeric nature of the chromatin, chromatin compaction, molecular crowding, phase separation, and anchoring to subnuclear compartments (Marshall et al., 1997; Spichal and Fabre, 2017; Caridi et al., 2018b). Two types of subdiffusive motions have been described: confined sub-diffusion and anomalous sub-diffusion.

\section{Confined Sub-diffusion}

When a chromosomal locus stays confined inside a sub-volume of the nucleus, its motion is called confined sub-diffusion (Figure 2A). The MSD exhibits a plateau (Marshall et al., 1997) and follows the equation:

$$
\operatorname{MSD}(\Delta t)=R_{\infty}^{2}\left(1-e^{-2 d D \Delta t / R_{\infty}^{2}}\right)+\varepsilon
$$

where $R_{\infty}$ is the measured plateau of the MSD, $D$ is the diffusion coefficient of the locus and $\varepsilon$ is the noise due to the experimental measurements. The confinement radius $(R c)$ of the motion is given by the relation: $R_{c}=R_{\infty} \sqrt{(d+2) / d}$, where $d$ is the dimension of the motion. It refers to the radius of a sphere inside which the motion is contained. The MSD curve starts to bend at time $t_{c}=R_{c}^{2} /(2 \mathrm{dD})$, representing the characteristic equilibration time after which the effect of boundaries appears.

\section{Anomalous Sub-diffusion}

When the force or structure that restricts the motion is not a simple confinement but is modulated in time and space with scaling properties, the motion is called anomalous sub-diffusion (Barkai et al., 2012; Metzler et al., 2014) (Figure 2A). In this case, sub-diffusive loci are constrained, but, unlike confined loci, they can diffuse without boundary and thus reach further targets if given enough time. For sub-diffusive motion, the MSD exhibits a power law,

$$
\operatorname{MSD}(\Delta t)=A \Delta t^{\alpha}+\varepsilon
$$

where $\alpha$, the anomalous exponent, is smaller than 1 .

The anomalous exponent $\alpha$ is linked to the degree of recurrence of DNA exploration, i.e., the number of times a locus reiteratively scans neighboring regions before reaching a distant position (Ben-Avraham, 2000). When $\alpha$ is small, the locus recurrently explores the same environment for a long time, while a large $\alpha$ indicates that the locus is able to explore new environments often. The anomalous diffusion coefficient $A$ represents the amplitude of the motion; it is proportional to the diffusion coefficient only in the case of normal diffusion (when $\alpha=1$ ), which is rarely observed in biological systems (Barkai et al., 2012).

Experimental noise $\varepsilon$ can strongly affect MSD measurements also in the case of anomalous sub-diffusion. The exact formula to fit the MSD curves of anomalous diffusion, including localization accuracy, is given by the formula:

$$
\begin{aligned}
\operatorname{MSD}(\Delta t)=A \Delta t^{\alpha}+\sigma_{0}^{2}\left(1+\frac{A t_{E x p}^{\alpha}}{4 s_{0}^{2}}\right) & -\frac{A \alpha(1-\alpha) t_{E x p}^{2}}{12 \Delta t^{2}} \Delta t^{\alpha} \\
& -\frac{2 A}{(\alpha+1)(\alpha+2)}
\end{aligned}
$$

which has been calculated and used to characterize chromatin mobility at multiple time scales (Miné-Hattab et al., 2017).

\section{Directed Motion}

Recent studies of chromatin mobility in the context of DNA repair have revealed the existence of transient directed motion in living cells (Cho et al., 2014; Caridi et al., 2018a,b; Lamm et al., 2018; Oshidari et al., 2018) (Figure 2A). For directed motion, MSD values rapidly increase at higher time intervals, as follows:

$$
\operatorname{MSD}(\Delta t)=2 d D \Delta t+\nu^{2} \Delta t^{2}+\varepsilon
$$

where $D$ is the diffusion coefficient, $\nu$ is the velocity of the directed motion and $\varepsilon$ is the noise due to the experimental measurements.

\section{MSD ANALYSES REVEAL INCREASED NUCLEAR EXPLORATION OF DAMAGED AND UNDAMAGED CHROMATIN IN RESPONSE TO DSBS}

MSD analyses have been used to characterize the dynamics of repair sites in different contexts, from yeast to mammalian cells, deriving descriptive parameters like diffusion coefficient and confinement radius. In yeast, for example, MSD analyses of repair sites in response to $I S c e I$-induced breaks revealed that resected DSBs explore a nuclear volume up to ten times larger than before damage (Dion et al., 2012; Miné-Hattab and Rothstein, 2012). This response depends on resection, chromatin remodeling, checkpoint activation, the strand invasion component Rad51 (Oza et al., 2009; Dion et al., 2012; Miné-Hattab and Rothstein, 2012; Neumann et al., 2012; Horigome et al., 2014; Saad et al., 2014; Amitai et al., 2017; Miné-Hattab et al., 2017; Smith et al., 2018), and it has been linked to homology search (Dion et al., 2012; Miné-Hattab and Rothstein, 2012; Neumann et al., 2012; Miné-Hattab et al., 2017). This process is exceptionally efficient. For example, in S. cerevisiae, a single recipient locus and a single donor locus that share as little as $1.2 \mathrm{~kb}$ of homology will find each other in the $15,000 \mathrm{~kb}$ of genome, and engage in repair with $90 \%$ efficiency within $2 \mathrm{~h}$ after DSB formation (Aylon et al., 2003; Miné-Hattab and Rothstein, 2012). Increased nuclear exploration is more pronounced in diploid than in haploid cells (Dion et al., 2012; Miné-Hattab and Rothstein, 2012), potentially reflecting a more active search when the homologous partner is available (Mine-Hattab and Rothstein, 2013). Indeed, HR repair with the sister chromatid, which is kept in close proximity through cohesion, is not associated with extensive dynamics 
(Dion et al., 2012, 2013), further linking nuclear exploration with inter-homologous repair in yeast.

Importantly, studies in yeast revealed that undamaged loci also become more dynamic in response to damage, exploring a nuclear volume up to four times larger, and more DSBs induce larger nuclear exploration (Miné-Hattab and Rothstein, 2012; Seeber et al., 2013; Herbert et al., 2017; Lawrimore et al., 2017; Miné-Hattab et al., 2017). Changes in chromatin mobility are thus a general feature of the cellular response to DSBs affecting the whole genome. Experimental and theoretical studies suggest that changes in chromatin mobility of both damaged and undamaged loci increase the probability of contact between distant loci, thus promoting the kinetics of homologous pairing (Miné-Hattab and Rothstein, 2012; Guerin et al., 2016; MinéHattab et al., 2017; Amitai and Holcman, 2018).

Increased nuclear exploration of damaged sites during HR repair is also observed in mammalian and Drosophila cells (Chiolo et al., 2011; Krawczyk et al., 2012; Becker et al., 2014; Cho et al., 2014; Lottersberger et al., 2015; Ryu et al., 2015; Caridi et al., 2018a,b; Schrank et al., 2018). Studies of Rad52 foci in S phase of human cells revealed significant dynamics even when the sister chromatid is used as a template, and linked it to clustering of repair sites (Schrank et al., 2018) (Supplementary Table S1). Notably, in human cells NHEJ appears to operate more frequently than HR (Beucher et al., 2009), and does not require extensive movement (Krawczyk et al., 2012; Aymard et al., 2017; Schrank et al., 2018; Schrank and Gautier, 2019), except at unprotected telomeres (Dimitrova et al., 2008; Lottersberger et al., 2015). This might explain why repair focus dynamics have not been detected in early studies (Nelms et al., 1998; Soutoglou et al., 2007; Jakob et al., 2009). Further, studies in Drosophila cells treated with IR, revealed that both euchromatic and heterochromatic repair foci are mobilized (Caridi et al., 2018a), with the most extensive nuclear exploration associated with heterochromatic sites that relocalize to the nuclear periphery (Ryu et al., 2015; Caridi et al., 2018a).

Although the movement of undamaged sites has not been consistently tracked in these systems, the dynamics of other (undamaged) chromosomal loci (e.g., telomeres and centromeres) before and after damage suggest that global chromatin mobilization is also conserved (Lottersberger et al., 2015; Caridi et al., 2018a).

What promotes the dynamics of undamaged loci in response to DSBs? Different contributing mechanisms have been identified: (i) the release of structures that anchor chromosomal loci to the nuclear periphery, (ii) repair and checkpoint proteins; (iii) the transfer of cytoplasmic forces to the chromatin through the LINC complex; and (iv) global chromatin modifications. Specifically, anchoring of centromeres, telomeres and the nucleolus to the nuclear envelope provides constraints to the motion of interphase chromosomes in budding yeast, limiting chromosome dynamics (Berger et al., 2008; Therizols et al., 2010; Wong et al., 2012; Agmon et al., 2013; Verdaasdonk et al., 2013; Strecker et al., 2016; Lawrimore et al., 2017). Releasing telomere and centromere attachments reproduces chromatin mobility observed in response to DSBs (Strecker et al., 2016; Lawrimore et al., 2017). These studies also identified a Mecl-dependent phosphorylation of the kinetocore protein Cep3 as an essential player in global chromatin mobilization (Strecker et al., 2016). In addition to checkpoint kinases, Rad51 and Rad52 HR proteins are required to facilitate global chromatin dynamics (Seeber et al., 2013; Miné-Hattab et al., 2017; Smith et al., 2018, 2019). Further, studies in yeast and mammalian cells suggest that cytoplasmic actin and microtubules induce a global chromatin "shake-up" in response to DSB formation (Lottersberger et al., 2015; Spichal et al., 2016; Amitai et al., 2017; Lawrimore et al., 2017). Finally, intrinsic modifications of chromatin properties following DSBs, such as chromatin decondensation and changes in chromatin stiffness, appear to contribute to the global increase in chromatin dynamics. Global chromatin decondensation in response to DNA damage has been described across different model systems and likely results from histone modifications, chromatin remodeling, and histone loss (Ziv et al., 2006; Ayoub et al., 2008; Chiolo et al., 2011; Luijsterburg et al., 2012; Seeber et al., 2013; Strecker et al., 2016; Amitai et al., 2017; Hauer et al., 2017). These modifications might promote nuclear exploration by reducing chromatin compaction and increasing its flexibility. Additional studies applied numerical simulation of chromatin dynamics, mainly based on Rouse-like models (Arbona et al., 2017), to predict chromatin mobility in response to DSBs both at the damaged site and genome-wide (Herbert et al., 2017; Lawrimore et al., 2017; Miné-Hattab et al., 2017). For example, $\beta$-polymer modeling and simulations suggest that local chromatin expansion is sufficient to drive extrusion of the damage site from its local domain, affecting longer-range dynamics (Amitai et al., 2017). However, multi-scale tracking of chromatin (see: Multi-scale motion section, below) and polymer simulations also suggest the importance of chromatin stiffening in local and global chromatin dynamics (Herbert et al., 2017; Lawrimore et al., 2017; Miné-Hattab et al., 2017). This is potentially in contradiction with the role of chromatin relaxation in the same responses, and might reflect a different extent of relaxation/stiffening across distinct loci or time points following damage formation. Thus, more studies are needed to establish the relative contribution of chromatin stiffening and relaxation to increased chromatin exploration, toward an integrated model for damage-induced chromatin dynamics.

Of note, studies in yeast revealed that increased nuclear exploration does not correlate with higher speed of locus movement. In fact, the diffusion coefficient does not significantly change in response to damage, both at damaged and undamaged loci (Miné-Hattab and Rothstein, 2012; Mine-Hattab and Rothstein, 2013). In other words, changes in mobility allow chromatin to go further but not faster.

Overall, MSD analyses have been an invaluable tool for identifying damage-induced nuclear dynamics, revealing a significant increase of nuclear exploration in response to DSBs for both damaged and undamaged chromatin, and identifying several molecular mechanisms responsible for these dynamics.

\section{LIMITATIONS OF MSD ANALYSES}

Recent studies of chromatin trajectories in response to DNA damage revealed that MSD analyses also suffer from several limitations, and can even mask the existence of certain 
characteristics of the motion. First, MSDs are typically calculated as time-ensemble-averaged values over several trajectories to obtain a precise estimate of the parameters describing the motion (e.g., confinement radius and diffusion coefficient). This is in part to compensate for the location measurement errors mentioned above, and in part to enable the use of relatively short trajectories limited by photo-bleaching and photo-toxicity effects. However, averaging the behavior of several trajectories affects the ability to detect the differences between them, i.e., it does not account for heterogeneity across different cells and break sites. Second, MSD calculations assume that each site undergoes homogenous motion during the time of acquisition, which is rarely the case. For example: (i) repair sites can be transiently bound to the nuclear periphery or other nuclear structures; (ii) their motion can be different inside or outside phase separated domains; and (iii) directed motions can occur for limited time periods (Figure 2B, left). A locus can also undergo distinct diffusion regimes at different time scales, which simultaneously contribute to the motion of a particle. For example, a locus can exhibit a subdiffusive motion characterized by $A_{\text {micro }}$, in a region that itself moves with a diffusion coefficient $\mathrm{A}_{\text {macro }}$ (Figure 2B, right). Additionally, chromatin motion is not purely sub-diffusive even in the absence of DNA damage; studies in budding yeast (Heun et al., 2001) and Chinese hamster ovary cells (Levi et al., 2005) showed that chromatin undergoes confined random motion alternating with rare fast jumps that likely reflect rare events of active diffusion.

Accordingly, simulations of a particle moving with different types of motions: confined, directed, and a combination of confined and directed (mixed trajectory), show how MSD curves can mask the presence of directed motions (Figure 3 and Supplementary Movies S1-S3) (Bacher et al., 2004; Masedunskas et al., 2017). The simulation of a mixed trajectory accounts for asynchronous motion, where the starting point of directed motion and its duration is different for each particle as observed experimentally. While MSD curves for confined and directed motions display the expected shapes (Figures $4 \mathbf{A}, \mathbf{B}$ ), the MSD graph for mixed trajectories resembles that describing a subdiffusive confined motion (Figure 4C), confirming that the MSD approach is not suitable to describe heterogenous and asynchronous motions.

In the following sections, we will illustrate two major types of complex motions occurring in response to DNA damage (Figure 4), and we will discuss experimental approaches and analytical methods that enabled their characterization beyond simple MSD analyses.

\section{MIXED TRAJECTORIES}

A major question in the field of nuclear dynamics is whether repair focus motion is driven by active forces, or alternatively subdiffusive motions followed by anchoring to subnuclear structures are sufficient to generate these dynamics. Recent studies revealed the existence of directed motions in a context of mixed trajectories for at least some damage-induced responses (reviewed in Caridi et al., 2019).
First, IR-induced heterochromatic repair foci that relocalize to the nuclear periphery in Drosophila cells, are characterized by directed motion driven by transient nuclear actin filaments (F-actin) and myosins (Caridi et al., 2018a; Dialynas et al., 2019; See et al., 2020) (reviewed in Caridi et al., 2019) (Figure 4A, left). Repair foci slide along the filaments, and focus movement requires myosins' ability to walk along filaments, suggesting that nuclear F-actin provides "highways" for the relocalization of repair sites via myosin motors (Caridi et al., 2018a). Myosins and the actin nucleator Arp2/3 associate with the heterochromatin repair component Smc5/6 in response to damage, suggesting Smc5/6 as a physical link between heterochromatic repair sites and the motor system (Caridi et al., 2018a). Smc5/6 also recruits the myosin activator Unc45 to repair sites, inducing chromatin mobilization (Caridi et al., 2018a). Further, relocalization requires SUMOylation, checkpoint and resection proteins, similar to other relocalization pathways (Chiolo et al., 2011; Ryu et al., 2015, 2016; Amaral et al., 2017; Caridi et al., 2018a). Defective relocalization results in unrepaired or misrepaired DSBs, revealing the importance of this pathway for "safe" HR in heterochromatin (Chiolo et al., 2011; Ryu et al., 2015, 2016; Caridi et al., 2018a; Dialynas et al., 2019). Notably, in this context, directed motions primarily occur between the periphery of the heterochromatin domain [a distinct structure in Drosophila cells (Chiolo et al., 2011; Li et al., 2017)] and the nuclear periphery, which is where most nuclear actin filaments are organized (Caridi et al., 2018a). Directed motions typically last $24 \mathrm{~min}$, corresponding to the average time required for repair sites to reach the nuclear periphery and the average duration of nuclear actin filaments (Caridi et al., 2018a). However, time points coinciding with the initial movement of repair sites from inside the heterochromatin domain to its periphery are characterized by confined diffusion (Caridi et al., 2018a; Rawal et al., 2019), similar to the rest of undamaged heterochromatin that behaves like a phase separated domain (Larson et al., 2017; Strom et al., 2017). Time points following focus association with the nuclear periphery also display confined diffusion (Ryu et al., 2015; Caridi et al., 2018a; Rawal et al., 2019). In this context where directed motions alternate with diffusive motions, and initiate asynchronously in the population of foci, directed motions are not detected in a simple time-ensemble MSD analysis (Ryu et al., 2015; Caridi et al., 2018a,b) (Supplementary Table S1). Time points characterized by directed motions were identified using an analytical method that scans the trajectory of each focus at variable time windows and initiation times, and detects time windows in which MSD graphs displays upward curvature (Caridi et al., 2018a,b) (Supplementary Table S1). Isolating these time points also required imaging techniques that minimize cell movement and correct for modest rotational and translational motion of the nuclei (Amitai et al., 2017), removing a significant amount of noise from the system (Caridi et al., 2018b; See et al., 2020) (Supplementary Table S1). Additionally, given the long time span along which these motions occur, optimizing imaging conditions for long time imaging and sufficiently spaced time intervals is essential for their detection (Ryu et al., 2015; Caridi et al., 2018a,b; See et al., 2020). 


\section{Computer simulations of chromatin motion types}

\section{A Confined}

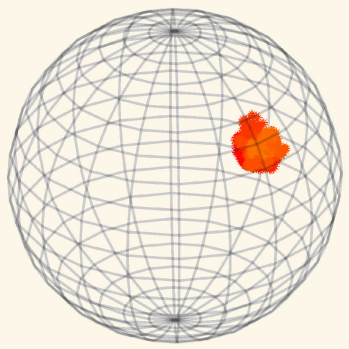

B Directed

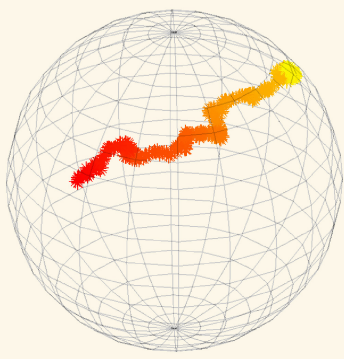

C Mixed

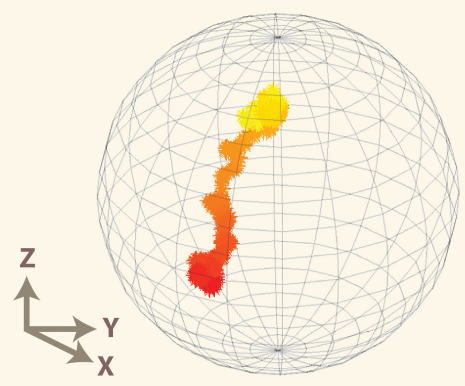

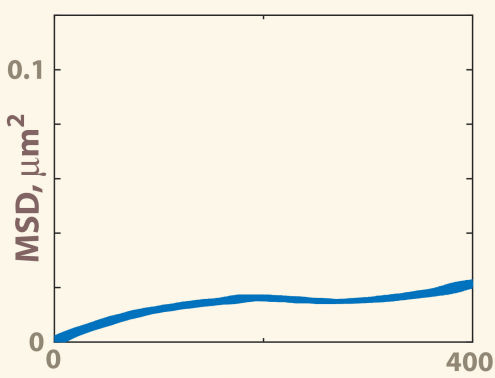

$\Delta \mathbf{t}$, sec

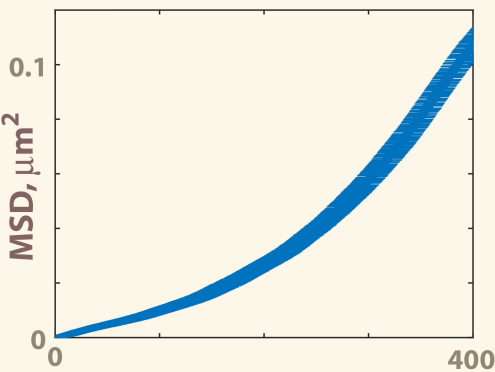

$\Delta \mathbf{t}, \sec$

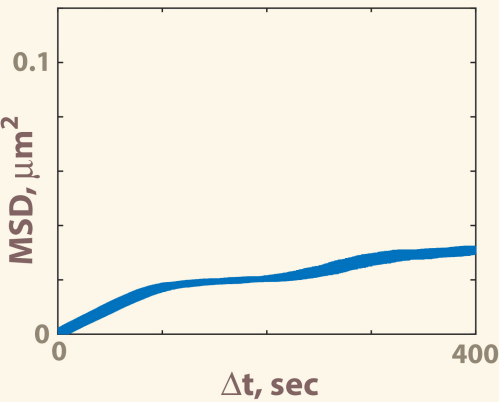

FIGURE 3 | Simulation of confined motion, directed motion and mixed trajectories. The motion of a particle in a sphere of $1 \mu \mathrm{m}$ radius was simulated using 1,000 iterations (Bacher et al., 2004; Masedunskas et al., 2017). Early timepoints are colored in red, late timepoints in yellow. (A) Example of a trajectory obtained by simulating a confined motion $\left(D=0.005 \mu \mathrm{m}^{2} / \mathrm{s}, \mathrm{Rc}=0.3 \mu \mathrm{m}\right.$ ) (see also corresponding Supplementary Movie S1). (B) Example of a trajectory obtained by simulating directed motion until the particle reaches the surface of the sphere $\left(D=0.005 \mu \mathrm{m}^{2} / \mathrm{s}, \nu=1 \mu \mathrm{m}\right)$ (see also corresponding Supplementary Movie S2). (C) Example of a mixed trajectory characterized by confined motion $\left(D=0.005 \mu \mathrm{m}^{2} / \mathrm{s}, \mathrm{Rc}=0.3 \mu \mathrm{m}\right)$ lasting 200 timepoints, followed by directed motion $\left(D=0.005 \mu \mathrm{m}^{2} / \mathrm{s}, \nu=1 \mu \mathrm{m}\right.$ for $\left.t=201-400\right)$ and confined motion for the last 600 time points $\left(D=0.005 \mu \mathrm{m}^{2} / \mathrm{s}\right.$, Rc $=0.3 \mu \mathrm{m}$. Time-ensemble MSDs were calculated over 10 trajectories. For panel (C), each trajectory is characterized by a different time point when the directed motion starts, and different duration of the directed motion (see also Supplementary Movie S3).

Notably, these studies also established that the average speed of focus motion associated with the relocalization of heterochromatic DSBs is not higher at time points characterized by directed motion relative to time points characterized by confined diffusion (Caridi et al., 2018a; Rawal et al., 2019). This is consistent with a model where actin filaments and motors do not increase motion speed. Rather, they provide directionality and counteract other forces that might limit the release of repair foci from the heterochromatin domain (e.g., chromatin compaction and/or phase separation) (Rawal et al., 2019).

Second, in a study currently in preprint, application of similar analysis methods identified short time points characterized by directed motions for damaged replication forks in human cells, which also correlate with the formation of nuclear actin filaments and the restart of stalled forks (Lamm et al., 2018). 


\section{A Complex motion of chromatin}

D. melanogaster

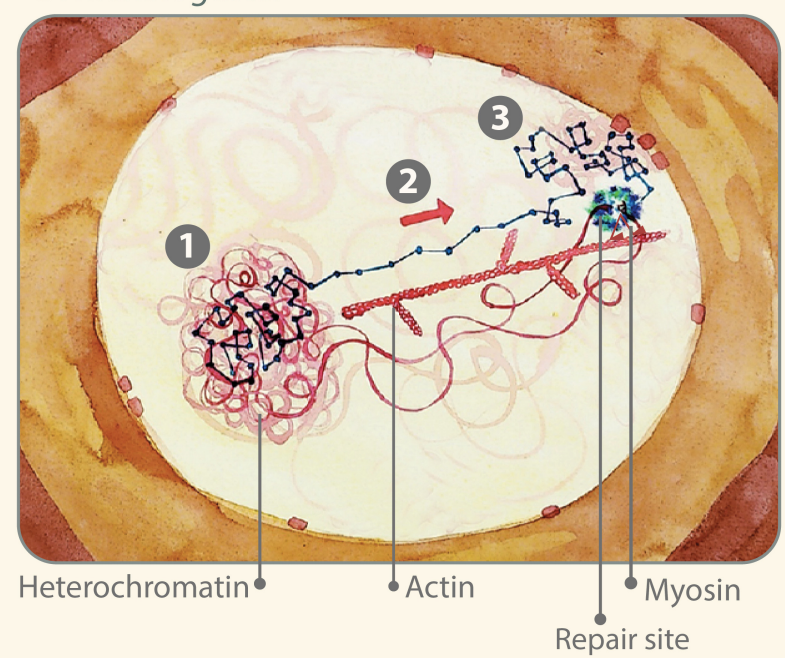

\section{S. cerevisiae}

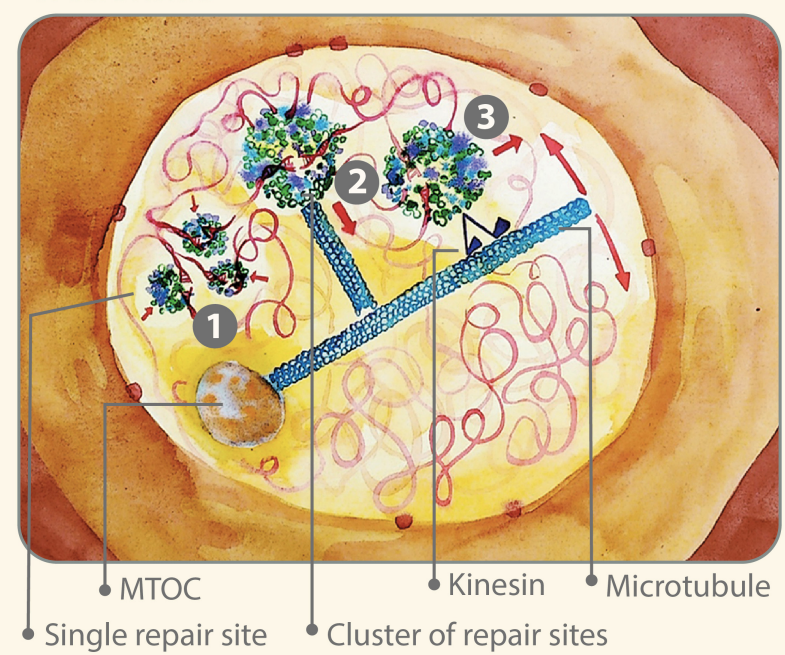

\section{B Multi-Scale Imaging}

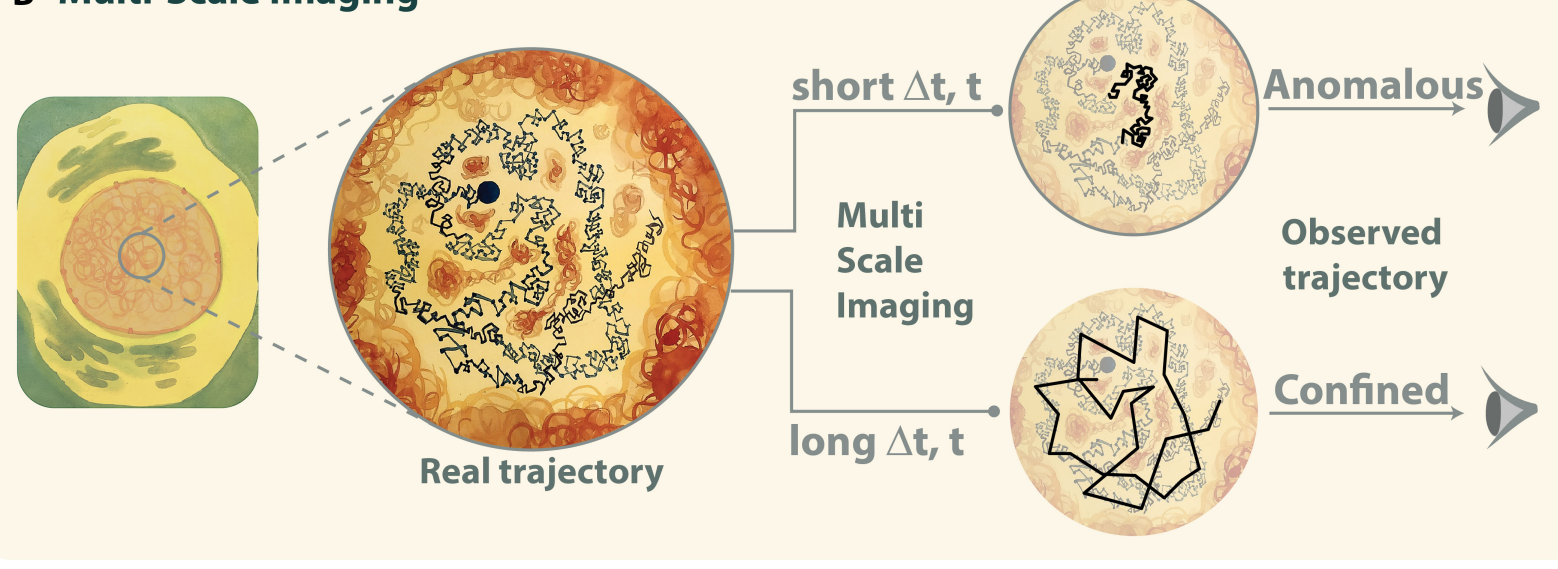

FIGURE 4 | (A) Illustration of examples of mixed trajectories. Left: in Drosophila cells, the motion of DSBs leaving the heterochromatin domain and reaching the nuclear periphery is characterized by: (1) confined diffusion inside the heterochromatin domain; (2) myosin-driven directed motion along actin filaments between the heterochromatin domain periphery and the nuclear periphery; and (3) confined diffusion at the nuclear periphery. Right: in budding yeast, repair sites: (1) cluster into larger foci; (2) are "captured"i by short microtubules; and (3) move by kinesin-driven directed motions along long nuclear microtubules that pivot around the microtubule organizing center (MTOC). (B) Illustration of multi-scale motion. Chromatin imaging at different time scales reveals anomalous diffusion at short time intervals $(\Delta t)$ and confined diffusion at longer time intervals. It is important to keep in mind that experiments reveal only the mobility of molecules accessible with the specific imaging conditions used during the acquisition. Thus, different imaging settings can shed light on different diffusive behaviors, which are visible only at certain time scales. Illustration by Olga Markova.

Third, directed motions have been detected during homology search for HR repair of telomeres in ALT human cells (Cho et al., 2014), which might also potentially include C-circles released from telomeres (Henson et al., 2009; Schrank and Gautier, 2019; Zhang et al., 2019). In this case, time-ensembleaverage MSD graphs were characterized by $\alpha>1$ when calculated at selected time points preceding telomere-telomere association for ALT repair, effectively limiting the analysis to time points when the motion is homogeneous (Supplementary Table S1).
Fourth, directed motions have been described for subtelomeric DSBs repaired by the HR sub-pathway breakinduced replication (BIR) in S. cerevisiae (Oshidari et al., 2018) (Figure 4A, right). These damage sites move along a single nuclear microtubule but directed motions are not easily detectable using canonical MSD analyses because of two major confounding effects: (i) DSB movement along microtubules is transient; and (ii) microtubules pivot around the microtubule organizing center (MTOC), resulting in non-linear directed motions (Oshidari et al., 2018). In this case, directed motions 
were identified by directional change distribution (DCD) analysis, which measures changes in the angle of a trajectory and can reveal broader motion profiles by increasing the temporal coarse graining (Oshidari et al., 2018) (reviewed in Oshidari et al., 2019b) (Supplementary Table S1). This study also identified a role for Kar3 in kinesin-dependent directed motions and BIR completion (Oshidari et al., 2018). Notably, loss of Kar3 does not affect the average speed of motion (Oshidari et al., 2018), suggesting that also in this context, filaments and motors have a role in providing directionality to the repair site motion rather than affecting speed. In addition to these functions, short nuclear microtubules have been proposed to generate a flow that facilitates clustering of repair foci, and additional short filaments departing from these clusters promote the capturing of repair centers by the main microtubule (Oshidari et al., 2019a).

Fifth, application of the DCD analysis also identified directed motions for persistent DSBs that move to the nuclear periphery in budding yeast (Oshidari et al., 2018), reverting the previous conclusion that these are characterized by diffusive motion followed by nuclear periphery anchoring (Amitai et al., 2017).

Finally, Arp2/3 and nuclear actin polymerization contributes to repair focus clustering and HR repair in Drosophila and mammalian cells (Caridi et al., 2018a; Schrank et al., 2018), and short actin filaments travel with repair foci in human cells (Schrank et al., 2018), suggesting a direct role of these structures in mobilizing damage sites. While directed motions have not been directly investigated in this context, and myosins do not seem to be involved (Caridi et al., 2018a), the requirement of nuclear filaments suggest that directed motions might also contribute to these dynamics (Caridi et al., 2019).

It is worth noting that, in addition to heterochromatin, other membraneless -or phase separated-compartments exist in the nucleus, including nucleoli and repair foci per se (Altmeyer et al., 2015; Frottin et al., 2019; Kilic et al., 2019; Min et al., 2019; Singatulina et al., 2019) (reviewed in Mine-Hattab and Taddei, 2019; Rawal et al., 2019), which can affect the dynamics of repair foci at different levels. Phase separation of a nuclear domain might promote diffusion of repair sites inside the domain, while limiting release from the domain due to surface tension (Hyman et al., 2014). Phase separation properties of repair components might also contribute to the clustering of repair foci into larger structures, promoting local dynamics (Altmeyer et al., 2015; Kilic et al., 2019; Oshidari et al., 2019a). Notably, as repair sites move from one domain to another, their motion is likely to change properties exhibiting successive diffusion regimes, which cannot be detected with time-ensemble MSD analyses (Figure $\mathbf{4 A}$, right). In all these cases, dedicated analytical methods should be applied to characterize the diffusion regimes involved.

Further, damage-induced nuclear dynamics can occur in the context of a dynamic nucleus, which adds rotational motion to the system. In yeast, removal of nuclear rotations via Latrunculin treatment enabled the identification of modes of diffusion that are otherwise masked by the nuclear rotational movement (Amitai et al., 2017). In mouse and Drosophila cells, these rotational movements were corrected by registering the nuclei relative to repair foci prior to tracking repair sites to establish repair locus trajectories (Ryu et al., 2015; Caridi et al., 2018a,b; See et al., 2020) (Supplementary Table S1).

These studies point to the importance of applying dedicated imaging approaches, image processing methods, and analytical tools to identify directed motions. They also suggest that nuclear structures and motors contribute to repositioning repair sites in more situations than initially thought, including where diffusive motions appear to prevail. More studies are needed to identify repair contexts relying on directed movements and the structural/motor components mediating these dynamics, and more methods need to be developed to account for different types of mixed trajectories.

\section{MULTI-SCALE MOTION}

Chromatin presents several levels of organization, which translates into different scales of chromatin mobility (MineHattab and Darzacq, 2018). These different modes of diffusion can be unraveled by imaging the chromatin at different timescales (Figure 4B and Supplementary Table S1). For example, in the absence of DNA damage, chromatin undergoes anomalous diffusion when observed at short time intervals (10-ms to 1-s) (Maeshima et al., 2010; Weber et al., 2010; Burnecki et al., 2012; Hajjoul et al., 2013; Lucas et al., 2014; Backlund et al., 2015; Amitai et al., 2017; Miné-Hattab et al., 2017). However, at longer time scales, MSD exhibits a plateau characteristic of confined diffusion, consistent with the chromatin remaining confined inside a sub-volume of the nucleus (Marshall et al., 1997; Heun et al., 2001; Maeshima et al., 2010; Masui et al., 2011; Miné-Hattab and Rothstein, 2012; Backlund et al., 2015).

Several recent studies applied multi scale imaging to characterize chromatin mobility in response to DSBs. Increased exploration of the nuclear space is detected in response to I-SceI-induced DSBs when imaging is done at $1.5 \mathrm{~s}$ or longer time intervals (Dion et al., 2012; Miné-Hattab and Rothstein, 2012). However, remarkably, imaging at $100 \mathrm{~ms}$ time intervals or faster reveals lower mobility of the damaged site relative to undamaged conditions (Miné-Hattab et al., 2017). Given that a shorter time scale for data collection investigates chromatin motion on a smaller temporal and spatial scale, the low mobility observed at short time scales reflects reduced local mobility of the cut site (Miné-Hattab et al., 2017). These dynamics can be modeled assuming that chromatin persistence length (a measure of the bending stiffness of a polymer) globally increases (Herbert et al., 2017; Miné-Hattab et al., 2017). At the damaged sites, such response likely results from the recruitment of the repair machinery that increases chromatin stiffness (Mine et al., 2007). Accordingly, reduced local mobility has been associated with resected DNA and requires Rad51 (Saad et al., 2014; Miné-Hattab et al., 2017).

The reduced mobility detected at lower time scales also characterizes undamaged chromatin, consistent with a global increase in chromatin stiffness that spreads beyond the damaged 
loci (Herbert et al., 2017; Miné-Hattab et al., 2017). This might depend on $\mathrm{H} 2 \mathrm{~A}$ phosphorylation, which spreads for kilobases to megabases from the cut site (Rogakou et al., 1999), and introduces negative charges into the chromatin (Herbert et al., 2017). As a consequence of a global increase in chromatin stiffness, intrachromosomal loci become more distant and their dynamics change, as observed experimentally in yeast (Herbert et al., 2017; Miné-Hattab et al., 2017).

It has been proposed that increased rigidity of the chromatin facilitates the movement of the cut site through the dense nucleoplasm (Miné-Hattab et al., 2017). In other words, a stiffer chromatin (with more rigidity associated with the break site) would enable resected DNA to navigate through adjacent obstacles more efficiently, thus allowing it to reach farther targets. The stiffer chromatin would act like a needle to help move damaged DNA through the chromatin mesh, likened to a "ball of yarn" (Miné-Hattab et al., 2017). Of note, there is currently no method to directly measure chromatin flexibility in living cells. The two studies referred to here (Herbert et al., 2017; MinéHattab et al., 2017) use indirect methods to assess chromatin stiffness, by comparing conformation and dynamics of tagged chromosomal loci with polymer simulation.

These studies emphasize the importance of interrogating different spatiotemporal scales to understand chromatin motions, potentially revealing distinct dynamic processes and regulatory mechanisms. Additionally, more sophisticated and refined mathematical tools are necessary to account for the composite nature of chromatin motion, and for example to distinguish between the local diffusion of a locus in a region that itself moves with a different mode of diffusion.

\section{CONCLUSION AND PERSPECTIVES}

A large number of studies in the past decade have shown that DSBs trigger a larger exploration of the nucleus for damaged and undamaged chromatin sites, and this response is conserved from yeast to mammalian cells. Increasing chromatin confinement radius, or changing the nature of its motion, dramatically enhances the ability of a locus to sample neighboring DNA sequences during homology search. In addition to this response, recent studies have shown that chromatin motion is more complex than initially anticipated. Relocalization of repair sites via molecular motors typically results in mixed trajectories, where directed motions occur in alternation with subdiffusive regimes. Further, the transient directed movement of repair sites along oscillating structures (e.g., nuclear microtubules), nuclear flows, and phase separation of nuclear domains, add complexity to the trajectories. Additionally, distinct diffusion regimes typically occur at different time scales, likely reflecting different level of chromatin organization. A simple MSD analysis is not adapted for such composite motions, as it assumes a homogenous mode of diffusion during the acquisition. Additionally, timeensemble MSD analyses mix different type of motions that start asynchronously and occur for different durations. New analytical methods enabled the dissection of some of these dynamics. To reveal the existence of several diffusion regimes, multi-scale tracking, simulations, and mathematical models of complex motions need to be performed. The identification of several contexts where nuclear dynamics is dependent on nuclear actin filaments or microtubules, and characterized by short or long tracts of directed motions, suggests the existence of forces that drive the motion in more situations than initially thought. The development of new dedicated analytical methods started unlocking the door toward a deeper understanding of these dynamics, and the discovery of the molecular mechanisms responsible for their regulation. Nuclear dynamics facilitate DNA repair in different contexts, but nuclear exploration of damaged sequences is also responsible for chromosome rearrangements (Neumann et al., 2012; Roukos et al., 2013; Marcomini et al., 2018). Defects in relocalization pathways also result in genome instability (Torres-Rosell et al., 2007; Chiolo et al., 2011; Ryu et al., 2015, 2016; Su et al., 2015; Caridi et al., 2018a; Dialynas et al., 2019; Aguilera et al., 2020) (reviewed in Caridi et al., 2017; Caridi et al., 2019; Schrank and Gautier, 2019), and establishing the mechanisms responsible for these dynamics is a necessary step to understand how their misregulation contributes to cancer and other genome instability disorders.

\section{AUTHOR CONTRIBUTIONS}

Both authors equally contributed to this review and approved the submitted version.

\section{FUNDING}

This work was supported by NIH R01GM117376 and NSF Career 1751197 to IC and ANR (Agence Nationale pour la Recherche), the Labex DEEP (ANR-11-LABX-0044-DEEP and ANR-10-IDEX-0001-02 PSL), ANR-18-CE12-0015-03, the FRM (Fondation pour la Recherche Médicale), the PIC3i (Program Interdisciplinaire Curie), and the Q-Life program to JM-H and her team.

\section{ACKNOWLEDGMENTS}

We thank Scott Keagy and Mathias Heltberg for critical reading of the manuscript and Olga Markova for the artwork.

\section{SUPPLEMENTARY MATERIAL}

The Supplementary Material for this article can be found online at: https://www.frontiersin.org/articles/10.3389/fgene. 2020.00800/full\#supplementary-material 


\section{REFERENCES}

Agmon, N., Liefshitz, B., Zimmer, C., Fabre, E., and Kupiec, M. (2013). Effect of nuclear architecture on the efficiency of doublestrand break repair. Nat. Cell Biol. 15, 694-699. doi: 10.1038/ncb 2745

Aguilera, P., Whalen, J., Minguet, C., Churikov, D., Freudenreich, C., Simon, M. N., et al. (2020). The nuclear pore complex prevents sister chromatid recombination during replicative senescence. Nat. Commun. 11:160.

Altmeyer, M., Neelsen, K. J., Teloni, F., Pozdnyakova, I., Pellegrino, S., Grofte, M., et al. (2015). Liquid demixing of intrinsically disordered proteins is seeded by poly(ADP-ribose). Nat. Commun. 6:8088.

Amaral, N., Ryu, T., Li, X., and Chiolo, I. (2017). Nuclear Dynamics of Heterochromatin Repair. Trends Genet. 33, 86-100. doi: 10.1016/j.tig.2016. 12.004

Amitai, A., and Holcman, D. (2018). Encounter times of chromatin loci influenced by polymer decondensation. Phys. Rev. E 97:032417.

Amitai, A., Seeber, A., Gasser, S. M., and Holcman, D. (2017). Visualization of Chromatin Decompaction and Break Site Extrusion as Predicted by Statistical Polymer Modeling of Single-Locus Trajectories. Cell Rep. 18, 1200-1214. doi: 10.1016/j.celrep.2017.01.018

Arbona, J. M., Herbert, S., Fabre, E., and Zimmer, C. (2017). Inferring the physical properties of yeast chromatin through Bayesian analysis of whole nucleus simulations. Genome Biol. 18:81.

Aten, J. A., Stap, J., Krawczyk, P. M., van Oven, C. H., Hoebe, R. A., Essers, J., et al. (2004). Dynamics of DNA double-strand breaks revealed by clustering of damaged chromosome domains. Science 303, 92-95. doi: 10.1126/science. 1088845

Aylon, Y., Liefshitz, B., Bitan-Banin, G., and Kupiec, M. (2003). Molecular dissection of mitotic recombination in the yeast Saccharomyces cerevisiae. Mol. Cell Biol. 23, 1403-1417. doi: 10.1128/mcb.23.4.1403-1417.2003

Aymard, F., Aguirrebengoa, M., Guillou, E., Javierre, B. M., Bugler, B., Arnould, C., et al. (2017). Genome-wide mapping of long-range contacts unveils clustering of DNA double-strand breaks at damaged active genes. Nat. Struct. Mol. Biol. 24, 353-361. doi: 10.1038/nsmb.3387

Ayoub, N., Jeyasekharan, A. D., Bernal, J. A., and Venkitaraman, A. R. (2008). HP1beta mobilization promotes chromatin changes that initiate the DNA damage response. Nature 453, 682-686. doi: 10.1038/nature06875

Bacher, C. P., Reichenzeller, M., Athale, C., Herrmann, H., and Eils, R. (2004). 4$\mathrm{D}$ single particle tracking of synthetic and proteinaceous microspheres reveals preferential movement of nuclear particles along chromatin - poor tracks. BMC Cell Biol. 5:45. doi: 10.1186/1471-2121-5-45

Backlund, M. P., Joyner, R., and Moerner, W. E. (2015). Chromosomal locus tracking with proper accounting of static and dynamic errors. Phys. Rev. E Stat. Nonlin. Soft. Matter. Phys. 91:062716.

Barkai, E., Garini, Y., and Metzler, R. (2012). Strange kinetics of single molecules in living cells. Phys. Today 65, 29-35. doi: 10.1063/pt.3.1677

Becker, A., Durante, M., Taucher-Scholz, G., and Jakob, B. (2014). ATM alters the otherwise robust chromatin mobility at sites of DNA double-strand breaks (DSBs) in human cells. PLoS One 9:e92640. doi: 10.1371/journal.pone.0092640

Bekker-Jensen, S., Lukas, C., Kitagawa, R., Melander, F., Kastan, M. B., Bartek, J., et al. (2006). Spatial organization of the mammalian genome surveillance machinery in response to DNA strand breaks. J. Cell Biol. 173, 195-206. doi: $10.1083 /$ jcb. 200510130

Ben-Avraham, D. H. (2000). Diffusion and Reactions in Fractals and Disordered Systems. Cambridge: Cambridge University Press.

Berger, A. B., Cabal, G. G., Fabre, E., Duong, T., Buc, H., Nehrbass, U., et al. (2008). High-resolution statistical mapping reveals gene territories in live yeast. Nat. Methods 5, 1031-1037. doi: 10.1038/nmeth.1266

Beucher, A., Birraux, J., Tchouandong, L., Barton, O., Shibata, A., Conrad, S., et al. (2009). ATM and Artemis promote homologous recombination of radiationinduced DNA double-strand breaks in G2. EMBO J. 28, 3413-3427. doi: 10. 1038/emboj.2009.276

Brinkman, E. K., Chen, T., de Haas, M., Holland, H. A., Akhtar, W., and van Steensel, B. (2018). Kinetics and Fidelity of the Repair of Cas9-Induced DoubleStrand DNA Breaks. Mol. Cell 70, 801-813.e6. doi: 10.1016/j.molcel.2018. 04.016
Bronstein, I., Israel, Y., Kepten, E., Mai, S., Shav-Tal, Y., Barkai, E., et al. (2009). Transient anomalous diffusion of telomeres in the nucleus of mammalian cells. Phys. Rev. Lett. 103:018102.

Burnecki, K., Kepten, E., Janczura, J., Bronshtein, I., Garini, Y., and Weron, A. (2012). Universal algorithm for identification of fractional Brownian motion. A case of telomere subdiffusion. Biophys. J. 103, 1839-1847. doi: 10.1016/j.bpj. 2012.09.040

Bystricky, K., Laroche, T., van Houwe, G., Blaszczyk, M., and Gasser, S. M. (2005). Chromosome looping in yeast: telomere pairing and coordinated movement reflect anchoring efficiency and territorial organization. J. Cell Biol. 168, 375-387.

Caridi, C. P., D’Agostino, C., Ryu, T., Zapotoczny, G., Delabaere, L., Li, X., et al. (2018a). Nuclear F-actin and myosins drive relocalization of heterochromatic breaks. Nature 559, 54-60. doi: 10.1038/s41586-0180242-8

Caridi, C. P., Delabaere, L., Tjong, H., Hopp, H., Das, D., Alber, F., et al. (2018b). Quantitative Methods to Investigate the 4D Dynamics of Heterochromatic Repair Sites in Drosophila Cells. Methods Enzymol. 601, 359-389. doi: 10.1016/ bs.mie.2017.11.033

Caridi, C. P., Plessner, M., Grosse, R., and Chiolo, I. (2019). Nuclear actin filaments in DNA repair dynamics. Nat. Cell Biol. 21, 1068-1077. doi: 10.1038/s41556019-0379-1

Caridi, P. C., Delabaere, L., Zapotoczny, G., and Chiolo, I. (2017). And yet, it moves: nuclear and chromatin dynamics of a heterochromatic double-strand break. Philos. Trans. R. Soc. Lond. B Biol. Sci. 372:20160291. doi: 10.1098/rstb. 2016.0291

Caron, P., Choudjaye, J., Clouaire, T., Bugler, B., Daburon, V., Aguirrebengoa, M., et al. (2015). Non-redundant Functions of ATM and DNA-PKcs in Response to DNA Double-Strand Breaks. Cell Rep. 13, 1598-1609. doi: 10.1016/j.celrep. 2015.10.024

Chiolo, I., Minoda, A., Colmenares, S. U., Polyzos, A., Costes, S. V., and Karpen, G. H. (2011). Double-strand breaks in heterochromatin move outside of a dynamic HP1a domain to complete recombinational repair. Cell 144, 732-744. doi: 10.1016/j.cell.2011.02.012

Chiolo, I., Tang, J., Georgescu, W., and Costes, S. V. (2013). Nuclear dynamics of radiation-induced foci in euchromatin and heterochromatin. Mutat. Res. 750, 56-66. doi: 10.1016/j.mrfmmm.2013.08.001

Cho, N. W., Dilley, R. L., Lampson, M. A., and Greenberg, R. A. (2014). Interchromosomal homology searches drive directional ALT telomere movement and synapsis. Cell 159, 108-121. doi: 10.1016/j.cell.2014.08.030

Chung, D. K., Chan, J. N., Strecker, J., Zhang, W., Ebrahimi-Ardebili, S., Lu, T., et al. (2015). Perinuclear tethers license telomeric DSBs for a broad kinesinand NPC-dependent DNA repair process. Nat. Commun. 6:7742.

Churikov, D., Charifi, F., Eckert-Boulet, N., Silva, S., Simon, M. N., Lisby, M., et al. (2016). SUMO-Dependent Relocalization of Eroded Telomeres to Nuclear Pore Complexes Controls Telomere Recombination. Cell Rep. 15, 1242-1253. doi: 10.1016/j.celrep.2016.04.008

Clouaire, T., and Legube, G. (2019). A Snapshot on the Cis Chromatin Response to DNA Double-Strand Breaks. Trends Genet. 35, 330-345. doi: 10.1016/j.tig. 2019.02.003

Cohen, S., Puget, N., Lin, Y. L., Clouaire, T., Aguirrebengoa, M., Rocher, V., et al. (2018). Senataxin resolves RNA:DNA hybrids forming at DNA double-strand breaks to prevent translocations. Nat. Commun. 9:533.

Delabaere, L., and Chiolo, I. (2016). ReiNF4rcing repair pathway choice during cell cycle. Cell Cycle 15, 1182-1183. doi: 10.1080/15384101.2016.1159108

Delabaere, L., Ertl, H. A., Massey, D. J., Hofley, C. M., Sohail, F., and Bienenstock, E. J. (2017). Aging impairs double-strand break repair by homologous recombination in Drosophila germ cells. Aging Cell 16, 320-328. doi: 10.1111/ acel. 12556

Dialynas, G., Delabaere, L., and Chiolo, I. (2019). Arp2/3 and Unc45 maintain heterochromatin stability in Drosophila polytene chromosomes. Exp. Biol. Med. 244, 1362-1371. doi: 10.1177/1535370219862282

Dimitrova, N., Chen, Y. C., Spector, D. L., and de Lange, T. (2008). 53BP1 promotes non-homologous end joining of telomeres by increasing chromatin mobility. Nature 456, 524-528. doi: 10.1038/nature07433

Dion, V., and Gasser, S. M. (2013). Chromatin movement in the maintenance of genome stability. Cell 152, 1355-1364. doi: 10.1016/j.cell.2013.02.010 
Dion, V., Kalck, V., Horigome, C., Towbin, B. D., and Gasser, S. M. (2012). Increased mobility of double-strand breaks requires $\mathrm{Mec1}$, Rad9 and the homologous recombination machinery. Nat. Cell Biol. 14, 502-509. doi: 10. $1038 /$ ncb2465

Dion, V., Kalck, V., Seeber, A., Schleker, T., and Gasser, S. M. (2013). Cohesin and the nucleolus constrain the mobility of spontaneous repair foci. EMBO Rep. 14, 984-991. doi: 10.1038/embor.2013.142

Eckert-Boulet, N., Rothstein, R., and Lisby, M. (2011). Cell biology of homologous recombination in yeast. Methods Mol. Biol. 745, 523-536. doi: 10.1007/978-161779-129-1_30

Falk, M., Lukasova, E., Gabrielova, B., Ondrej, V., and Kozubek, S. (2007). Chromatin dynamics during DSB repair. Biochim. Biophys. Acta 1773, 15341545. doi: 10.1016/j.bbamcr.2007.07.002

Frottin, F., Schueder, F., Tiwary, S., Gupta, R., Korner, R., Schlichthaerle, T., et al. (2019). The nucleolus functions as a phase-separated protein quality control compartment. Science 365, 342-347. doi: 10.1126/science. aaw9157

Gehen, L. R., Gasser, S. M., and Dion, V. (2011). How Broken DNA Finds Its Template for Repair: A Computational Approach. Progre. Theor. Phys. Suppl. 191, 20-28.

Gennes, P. G. D. (1982). Kinetics of diffusion-controlled processes in dense polymer systems. II. Effects of entanglements. J. Chem. Phys. 76, 3322-3326. doi: $10.1063 / 1.443329$

Goodarzi, A. A., Noon, A. T., Deckbar, D., Ziv, Y., Shiloh, Y., Lobrich, M., et al. (2008). ATM signaling facilitates repair of DNA double-strand breaks associated with heterochromatin. Mol. Cell 31, 167-177. doi: 10.1016/j.molcel. 2008.05.017

Guénolé, A., and Legube, G. (2017). A Meeting at Risk: Unrepaired DSBs Go for Broke. Milton Park: Taylor \& Francis, 1-12.

Guerin, T., Levernier, N., Benichou, O., and Voituriez, R. (2016). Mean firstpassage times of non-Markovian random walkers in confinement. Nature 534, 356-359. doi: 10.1038/nature18272

Hajjoul, H., Mathon, J., Ranchon, H., Goiffon, I., Mozziconacci, J., Albert, B., et al. (2013). High-throughput chromatin motion tracking in living yeast reveals the flexibility of the fiber throughout the genome. Genome Res. 23, 1829-1838. doi: $10.1101 /$ gr.157008.113

Harding, S. M., Boiarsky, J. A., and Greenberg, R. A. (2015). ATM Dependent Silencing Links Nucleolar Chromatin Reorganization to DNA Damage Recognition. Cell Rep. 13, 251-259. doi: 10.1016/j.celrep.2015.08.085

Hauer, M. H., and Gasser, S. M. (2017). Chromatin and nucleosome dynamics in DNA damage and repair. Genes Dev. 31, 2204-2221. doi: 10.1101/gad. 307702.117

Hauer, M. H., Seeber, A., Singh, V., Thierry, R., Sack, R., Amitai, A., et al. (2017). Histone degradation in response to DNA damage enhances chromatin dynamics and recombination rates. Nat. Struct. Mol. Biol. 24, 99-107. doi: 10.1038/nsmb.3347

Henson, J. D., Cao, Y., Huschtscha, L. I., Chang, A. C., Au, A. Y., Pickett, H. A., et al. (2009). DNA C-circles are specific and quantifiable markers of alternativelengthening-of-telomeres activity. Nat. Biotechnol. 27, 1181-1185. doi: 10.1038/ nbt. 1587

Herbert, S., Brion, A., Arbona, J. M., Lelek, M., Veillet, A., Lelandais, B., et al. (2017). Chromatin stiffening underlies enhanced locus mobility after DNA damage in budding yeast. EMBO J. 36, 2595-2608. doi: 10.15252/embj. 201695842

Heun, P., Laroche, T., Shimada, K., Furrer, P., and Gasser, S. M. (2001). Chromosome dynamics in the yeast interphase nucleus. Science 294, 21812186. doi: 10.1126/science. 1065366

Horigome, C., Bustard, D. E., Marcomini, I., Delgoshaie, N., Tsai-Pflugfelder, M., Cobb, J. A., et al. (2016). PolySUMOylation by Siz2 and Mms21 triggers relocation of DNA breaks to nuclear pores through the Slx5/Slx8 STUbL. Genes Dev. 30, 931-945. doi: 10.1101/gad.277665.116

Horigome, C., Oma, Y., Konishi, T., Schmid, R., Marcomini, I., Hauer, M. H., et al. (2014). SWR1 and INO80 Chromatin Remodelers Contribute to DNA Double-Strand Break Perinuclear Anchorage Site Choice. Mol. Cell 55, 626-639. doi: 10.1016/j.molcel.2014.06.027

Horigome, C., Unozawa, E., Ooki, T., and Kobayashi, T. (2019). Ribosomal RNA gene repeats associate with the nuclear pore complex for maintenance after DNA damage. PLoS Genet. 15:e1008103. doi: 10.1371/journal.pgen.1008103
Hyman, A. A., Weber, C. A., and Julicher, F. (2014). Liquid-liquid phase separation in biology. Annu. Rev. Cell Dev. Biol. 30, 39-58.

Iacovoni, J. S., Caron, P., Lassadi, I., Nicolas, E., Massip, L., Trouche, D., et al. (2010). High-resolution profiling of gammaH2AX around DNA double strand breaks in the mammalian genome. EMBO J. 29, 1446-1457. doi: 10.1038/ emboj. 2010.38

Jakob, B., Splinter, J., Conrad, S., Voss, K. O., Zink, D., Durante, M., et al. (2011). DNA double-strand breaks in heterochromatin elicit fast repair protein recruitment, histone $\mathrm{H} 2 \mathrm{AX}$ phosphorylation and relocation to euchromatin. Nucleic Acids Res. 39, 6489-6499. doi: 10.1093/nar/ gkr230

Jakob, B., Splinter, J., Durante, M., and Taucher-Scholz, G. (2009). Live cell microscopy analysis of radiation-induced DNA double-strand break motion. Proc. Natl. Acad. Sci. U.S.A. 106, 3172-3177. doi: 10.1073/pnas.08109 87106

Janssen, A., Breuer, G. A., Brinkman, E. K., van der Meulen, A. I., Borden, S. V., van Steensel, B., et al. (2016). A single double-strand break system reveals repair dynamics and mechanisms in heterochromatin and euchromatin. Genes Dev. 30, 1645-1657. doi: 10.1101/gad.283028.116

Janssen, A., Colmenares, S. U., Lee, T., and Karpen, G. H. (2019). Timely doublestrand break repair and pathway choice in pericentromeric heterochromatin depend on the histone demethylase dKDM4A. Genes Dev. 33, 103-115. doi: $10.1101 / \operatorname{gad} .317537 .118$

Kalocsay, M., Hiller, N. J., and Jentsch, S. (2009). Chromosome-wide Rad51 spreading and SUMO-H2A.Z-dependent chromosome fixation in response to a persistent DNA double-strand break. Mol. Cell 33, 335-343. doi: 10.1016/j. molcel.2009.01.016

Khadaroo, B., Teixeira, M. T., Luciano, P., Eckert-Boulet, N., Germann, S. M., Simon, M. N., et al. (2009). The DNA damage response at eroded telomeres and tethering to the nuclear pore complex. Nat. Cell Biol. 11, 980-987. doi: $10.1038 / \mathrm{ncb} 1910$

Kilic, S., Lezaja, A., Gatti, M., Bianco, E., Michelena, J., Imhof, R., et al. (2019). Phase separation of 53BP1 determines liquid-like behavior of DNA repair compartments. EMBO J. 38:e101379.

Kong, X., Cruz, G. M. S., Silva, B. A., Wakida, N. M., Khatibzadeh, N., Berns, M. W., et al. (2018). Laser Microirradiation to Study In Vivo Cellular Responses to Simple and Complex DNA Damage. J. Vis. Exp. 131:56213.

Korsholm, L. M., Gal, Z., Lin, L., Quevedo, O., Ahmad, D. A., Dulina, E., et al. (2019). Double-strand breaks in ribosomal RNA genes activate a distinct signaling and chromatin response to facilitate nucleolar restructuring and repair. Nucleic Acids Res. 47, 8019-8035. doi: 10.1093/nar/ gkz518

Krawczyk, P. M., Borovski, T., Stap, J., Cijsouw, T., ten Cate, R., Medema, J. P., et al. (2012). Chromatin mobility is increased at sites of DNA double-strand breaks. J. Cell Sci. 125(Pt 9), 2127-2133. doi: 10.1242/jcs.08 9847

Kruhlak, M. J., Celeste, A., Dellaire, G., Fernandez-Capetillo, O., Müller, W. G., McNally, J. G., et al. (2006). Changes in chromatin structure and mobility in living cells at sites of DNA double-strand breaks. J. Cell Biol. 172, 823-834. doi: $10.1083 /$ jcb. 200510015

Kulashreshtha, M., Mehta, I. S., Kumar, P., and Rao, B. J. (2016). Chromosome territory relocation during DNA repair requires nuclear myosin 1 recruitment to chromatin mediated by Upsilon-H2AX signaling. Nucleic Acids Res. 44, 8272-8291. doi: 10.1093/nar/gkw573

Lamm, N., Masamsetti, V. P., Read, M. N., Biro, M., and Cesare, A. J. (2018). ATR and mTOR regulate F-actin to alter nuclear architecture and repair replication stress. bioRxiv [Preprint] doi: 10.1101/451708 v3.full

Larson, A. G., Elnatan, D., Keenen, M. M., Trnka, M. J., Johnston, J. B., Burlingame, A. L., et al. (2017). Liquid droplet formation by HPlalpha suggests a role for phase separation in heterochromatin. Nature 547, 236-240. doi: 10.1038/ nature 22822

Lawrimore, J., Barry, T. M., Barry, R. M., York, A. C., Friedman, B., Cook, D. M., et al. (2017). Microtubule dynamics drive enhanced chromatin motion and mobilize telomeres in response to DNA damage. Mol. Biol. Cell 28, 1701-1711. doi: 10.1091/mbc.e16-12-0846

Lee, C. S., Wang, R. W., Chang, H. H., Capurso, D., Segal, M. R., and Haber, J. E. (2016). Chromosome position determines the success 
of double-strand break repair. Proc. Natl. Acad. Sci. U.S.A. 113, E146E154.

Lemaitre, C., Grabarz, A., Tsouroula, K., Andronov, L., Furst, A., Pankotai, T., et al. (2014). Nuclear position dictates DNA repair pathway choice. Genes Dev. 28, 2450-2463. doi: 10.1101/gad.248369.114

Lenzken, S. C., Levone, B. R., Filosa, G., Antonaci, M., Conte, F., Kizilirmak, C., et al. (2019). FUS-dependent phase separation initiates double-strand break repair. bioRxiv [Preprint] doi: 10.1101/798884v1?rss=1

Levi, V., Ruan, Q., and Gratton, E. (2005). 3-D particle tracking in a two-photon microscope: application to the study of molecular dynamics in cells. Biophys. J. 88, 2919-2928. doi: 10.1529/biophysj.104.044230

Li, Q., Tjong, H., Li, X., Gong, K., Zhou, X. J., Chiolo, I., et al. (2017). The threedimensional genome organization of Drosophila melanogaster through data integration. Genome Biol. 18:145.

Lisby, M., Barlow, J. H., Burgess, R. C., and Rothstein, R. (2004). Choreography of the DNA damage response: spatiotemporal relationships among checkpoint and repair proteins. Cell 118, 699-713.

Lisby, M., Mortensen, U. H., and Rothstein, R. (2003). Colocalization of multiple DNA double-strand breaks at a single Rad52 repair centre. Nat. Cell Biol. 5, 572-577. doi: 10.1038/ncb997

Lottersberger, F., Karssemeijer, R. A., Dimitrova, N., and de Lange, T. (2015). 53BP1 and the LINC Complex Promote Microtubule-Dependent DSB Mobility and DNA Repair. Cell 163, 880-893. doi: 10.1016/j.cell.2015. 09.057

Lucas, J. S., Zhang, Y., Dudko, O. K., and Murre, C. (2014). 3D trajectories adopted by coding and regulatory DNA elements: first-passage times for genomic interactions. Cell 158, 339-352. doi: 10.1016/j.cell.2014.05.036

Luijsterburg, M. S., Lindh, M., Acs, K., Vrouwe, M. G., Pines, A., van Attikum, H., et al. (2012). DDB2 promotes chromatin decondensation at UV-induced DNA damage. J. Cell Biol. 197, 267-281. doi: 10.1083/jcb.2011 06074

Lukas, C., Bartek, J., and Lukas, J. (2005). Imaging of protein movement induced by chromosomal breakage: tiny 'local' lesions pose great 'global' challenges. Chromosoma 114, 146-154. doi: 10.1007/s00412-005-0011-y

Maeshima, K., Hihara, S., and Eltsov, M. (2010). Chromatin structure: does the 30nm fibre exist in vivo? Curr. Opin. Cell Biol. 22, 291-297. doi: 10.1016/j.ceb. 2010.03.001

Marcomini, I., Shimada, K., Delgoshaie, N., Yamamoto, I., Seeber, A., Cheblal, A., et al. (2018). Asymmetric Processing of DNA Ends at a Double-Strand Break Leads to Unconstrained Dynamics and Ectopic Translocation. Cell Rep. 24, 2614-2628e4.

Marnef, A., Finoux, A. L., Arnould, C., Guillou, E., Daburon, V., Rocher, V., et al. (2019). A cohesin/HUSH- and LINC-dependent pathway controls ribosomal DNA double-strand break repair. Genes Dev. 33, 1175-1190. doi: 10.1101/gad. 324012.119

Marshall, W. F., Straight, A., Marko, J. F., Swedlow, J., Dernburg, A., Belmont, A., et al. (1997). Interphase chromosomes undergo constrained diffusional motion in living cells. Curr. Biol. 7, 930-939. doi: 10.1016/s0960-9822(06)00412-x

Masedunskas, A., Chen, Y., Stussman, R., Weigert, R., and Mather, I. H. (2017). Kinetics of milk lipid droplet transport, growth, and secretion revealed by intravital imaging: lipid droplet release is intermittently stimulated by oxytocin. Mol. Biol. Cell 28, 935-946. doi: 10.1091/mbc.e16-11-0776

Masui, O., Bonnet, I., Baccon, P. Le, Brito, I., Pollex, T., Murphy, N., et al. (2011). Live-cell chromosome dynamics and outcome of $\mathrm{X}$ chromosome pairing events during ES cell differentiation. Cell 145, 447-458. doi: 10.1016/j.cell.2011.03.032

Mehta, I. S., Amira, M., Harvey, A. J., and Bridger, J. M. (2010). Rapid chromosome territory relocation by nuclear motor activity in response to serum removal in primary human fibroblasts. Genome Biol. 11:R5.

Metzler, R., Jeon, J. H., Cherstvy, A. G., and Barkai, E. (2014). Anomalous diffusion models and their properties: non-stationarity, non-ergodicity, and ageing at the centenary of single particle tracking. Phys. Chem. Chem. Phys. 16, 24128-24164. doi: $10.1039 / \mathrm{c} 4 \mathrm{cp} 03465 \mathrm{a}$

Michalet, X. (2010). Mean square displacement analysis of single-particle trajectories with localization error: brownian motion in an isotropic medium. Phys. Rev. E Stat. Nonlin Soft. Matter. Phys. 82(4 Pt 1):041914.

Michalet, X., and Berglund, A. J. (2012). Optimal diffusion coefficient estimation in single-particle tracking. Phys. Rev. E Stat. Nonlin. Soft. Matter. Phys. 85(6 Pt 1):061916.
Min, J., Wright, W. E., and Shay, J. W. (2019). Clustered telomeres in phaseseparated nuclear condensates engage mitotic DNA synthesis through BLM and RAD52. Genes Dev. 33, 814-827. doi: 10.1101/gad.324905.119

Mine, J., Disseau, L., Takahashi, M., Cappello, G., Dutreix, M., and Viovy, J. L. (2007). Real-time measurements of the nucleation, growth and dissociation of single Rad51-DNA nucleoprotein filaments. Nucleic Acids Res. 35, 7171-7187. doi: 10.1093/nar/gkm752

Mine-Hattab, J., and Darzacq, X. (2018). Dynamique de la chromatine en réponse aux dommages de l'ADN. Med. Sci. 34, 778-781. doi: 10.1051/medsci/2018214

Miné-Hattab, J., Recamier, V., Izeddin, I., Rothstein, R., and Darzacq, X. (2017). Multi-scale tracking reveals scale-dependent chromatin dynamics after DNA damage. Mol. Biol. Cell 28, 3323-3332. doi: 10.1091/mbc.e17-05-0317

Miné-Hattab, J., and Rothstein, R. (2012). Increased chromosome mobility facilitates homology search during recombination. Nat. Cell Biol. 14, 510-517. doi: $10.1038 / \mathrm{ncb} 2472$

Mine-Hattab, J., and Rothstein, R. (2013). DNA in motion during double-strand break repair. Trends Cell Biol. 23, 529-536.

Mine-Hattab, J., and Taddei, A. (2019). Physical principles and functional consequences of nuclear compartmentalization in budding yeast. Curr. Opin. Cell Biol. 58, 105-113. doi: 10.1016/j.ceb.2019.02.005

Nagai, S., Dubrana, K., Tsai-Pflugfelder, M., Davidson, M. B., Roberts, T. M., Brown, G. W., et al. (2008). Functional targeting of DNA damage to a nuclear pore-associated SUMO-dependent ubiquitin ligase. Science 322, 597-602. doi: $10.1126 /$ science. 1162790

Nelms, B. E., Maser, R. S., MacKay, J. F., Lagally, M. G., and Petrini, J. H. (1998). In situ visualization of DNA double-strand break repair in human fibroblasts. Science 280, 590-592. doi: 10.1126/science.280.5363.590

Neumaier, T., Swenson, J., Pham, C., Polyzos, A., Lo, A. T., Yang, P., et al. (2012). Evidence for formation of DNA repair centers and dose-response nonlinearity in human cells. Proc. Natl. Acad. Sci. U.S.A. 109, 443-448. doi: 10.1073/pnas. 1117849108

Neumann, F. R., Dion, V., Gehlen, L. R., Tsai-Pflugfelder, M., Schmid, R., Taddei, A., et al. (2012). Targeted INO80 enhances subnuclear chromatin movement and ectopic homologous recombination. Genes Dev. 26, 369-383. doi: 10.1101/ gad.176156.111

Oshidari, R., Huang, R., Medghalchi, M., Tse, E. Y. W., Ashgriz, N., Lee, H. O., et al. (2019a). DNA repair by Rad52 liquid droplets. bioRxiv [Preprint] doi: $10.1101 / 768119 \mathrm{v} 1$

Oshidari, R., Mekhail, K., and Seeber, A. (2019b). Mobility and Repair of Damaged DNA: random or Directed?. Trends Cell Biol. 30, 144-156. doi: 10.1016/j.tcb. 2019.11.003

Oshidari, R., Strecker, J., Chung, D. K. C., Abraham, K. J., Chan, J. N. Y., Damaren, C. J., et al. (2018). Nuclear microtubule filaments mediate non-linear directional motion of chromatin and promote DNA repair. Nat. Commun. 9:2567.

Oswald, F., Baa, L. M., Bollen, E. Y. J., and Peterman, E. J. (2014). Imaging and quantification of trans-membrane protein diffusion in living bacteria. Phys. Chem. Chem. Phys. 16, 12625-12634. doi: 10.1039/c4cp00299g

Oza, P., Jaspersen, S. L., Miele, A., Dekker, J., and Peterson, C. L. (2009). Mechanisms that regulate localization of a DNA double-strand break to the nuclear periphery. Genes Dev. 23, 912-927. doi: 10.1101/gad.17 82209

Pessina, F., Giavazzi, F., Yin, Y., Gioia, U., Vitelli, V., Galbiati, A., et al. (2019). Functional transcription promoters at DNA double-strand breaks mediate RNA-driven phase separation of damage-response factors. Nat. Cell Biol. 21, 1286-1299. doi: 10.1038/s41556-019-0392-4

Rawal, C. C., Caridi, C. P., and Chiolo, I. (2019). Actin' between phase separated domains for heterochromatin repair. DNA Repair 81:102646. doi: 10.1016/j. dnarep.2019.102646

Reynolds, P., Botchway, S. W., Parker, A. W., and O'Neill, P. (2013). Spatiotemporal dynamics of DNA repair proteins following laser microbeam induced DNA damage - when is a DSB not a DSB? Mutat. Res. 756, 14-20. doi: 10.1016/j. mrgentox.2013.05.006

Richardson, C. D., Kazane, K. R., Feng, S. J., Zelin, E., Bray, N. L., and Schafer, A. J. (2018). CRISPR-Cas9 genome editing in human cells occurs via the Fanconi anemia pathway. Nat. Genet. 50, 1132-1139. doi: 10.1038/s41588-0180174-0

Richardson, C. D., Ray, G. J., DeWitt, M. A., Curie, G. L., and Corn, J. E. (2016). Enhancing homology-directed genome editing by catalytically active 
and inactive CRISPR-Cas9 using asymmetric donor DNA. Nat. Biotechnol. 34, 339-344. doi: 10.1038/nbt.3481

Robinett, C. C., Straight, A., Li, G., Willhelm, C., Sudlow, G., Murray, A., et al. (1996). In vivo localization of DNA sequences and visualization of large-scale chromatin organization using lac operator/repressor recognition. J. Cell Biol. 135(6 Pt 2), 1685-1700. doi: 10.1083/jcb.135.6.1685

Rogakou, E. P., Boon, C., Redon, C., and Bonner, W. M. (1999). Megabase chromatin domains involved in DNA double-strand breaks in vivo. J. Cell Biol. 146, 905-916. doi: 10.1083/jcb.146.5.905

Roukos, V., Voss, T. C., Schmidt, C. K., Lee, S., Wangsa, D., and Misteli, T. (2013). Spatial dynamics of chromosome translocations in living cells. Science 341, 660-664. doi: 10.1126/science. 1237150

Ryu, T., Bonner, M. R., and Chiolo, I. (2016). Cervantes and Quijote protect heterochromatin from aberrant recombination and lead the way to the nuclear periphery. Nucleus 7, 485-497. doi: 10.1080/19491034.2016.1239683

Ryu, T., Spatola, B., Delabaere, L., Bowlin, K., Hopp, H., Kunitake, R., et al. (2015). Heterochromatic breaks move to the nuclear periphery to continue recombinational repair. Nat. Cell Biol. 17, 1401-1411. doi: 10.1038/ncb 3258

Saad, H., Gallardo, F., Dalvai, M., Tanguy-le-Gac, N., Lane, D., and Bystricky, K. (2014). DNA dynamics during early double-strand break processing revealed by non-intrusive imaging of living cells. PLoS Genet. 10:e1004187. doi: 10.1371/ journal.pgen.1004187

Schrank, B., and Gautier, J. (2019). Assembling nuclear domains: lessons from DNA repair. J. Cell Biol. 218, 2444-2455. doi: 10.1083/jcb.20190 4202

Schrank, B. R., Aparicio, T., Li, Y., Chang, W., Chait, B. T., Gundersen, G. G., et al. (2018). Nuclear ARP2/3 drives DNA break clustering for homology-directed repair. Nature 559, 61-66. doi: 10.1038/s41586-018-0237-5

See, C., Arya, D., Lin, E., and Chiolo, I. (2020). Live cell imaging of nuclear actin filaments and repair foci in Drosophila and mouse cells. Methods Mol. Biol. 7:e27900v1.

Seeber, A., Dion, V., and Gasser, S. M. (2013). Checkpoint kinases and the INO80 nucleosome remodeling complex enhance global chromatin mobility in response to DNA damage. Genes Dev. 27, 1999-2008. doi: 10.1101/gad.222992. 113

Singatulina, A. S., Hamon, L., Sukhanova, M. V., Desforges, B., Joshi, V., Bouhss, A., et al. (2019). PARP-1 Activation Directs FUS to DNA Damage Sites to Form PARG-Reversible Compartments Enriched in Damaged DNA. Cell Rep. 27, 1809-1821.e5.

Singleton, B. K., Griffin, C. S., and Thacker, J. (2002). Clustered DNA damage leads to complex genetic changes in irradiated human cells. Cancer Res. 62, 6263-6269.

Smith, M. J., Bryant, E. E., Joseph, F. J., and Rothstein, R. (2019). DNA damage triggers increased mobility of chromosomes in G1-phase cells. Mol. Biol. Cell 30, 2620-2625. doi: 10.1091/mbc.e19-08-0469

Smith, M. J., Bryant, E. E., and Rothstein, R. (2018). Increased chromosomal mobility after DNA damage is controlled by interactions between the recombination machinery and the checkpoint. Genes Dev. 32, 1242-1251. doi: 10.1101/gad.317966.118

Soutoglou, E., Dorn, J. F., Sengupta, K., Jasin, M., Nussenzweig, A., Ried, T., et al. (2007). Positional stability of single double-strand breaks in mammalian cells. Nat. Cell Biol. 9, 675-682. doi: 10.1038/ncb1591

Spichal, M., Brion, A., Herbert, S., Cournac, A., Marbouty, M., Zimmer, C., et al. (2016). Evidence for a dual role of actin in regulating chromosome organization and dynamics in yeast. J. Cell Sci. 129, 681-692. doi: 10.1242/jcs.17 5745

Spichal, M., and Fabre, E. (2017). The emerging role of the cytoskeleton in chromosome dynamics. Front. Genet. 8:60. doi: 10.3389/fgene.2017.00060

Strecker, J., Gupta, G. D., Zhang, W., Bashkurov, M., Landry, M. C., Pelletier, L., et al. (2016). DNA damage signalling targets the kinetochore to promote chromatin mobility. Nat. Cell Biol. 18, 281-290. doi: 10.1038/ncb3308

Strom, A. R., Emelyanov, A. V., Mir, M., Fyodorov, D. V., Darzacq, X., and Karpen, G. H. (2017). Phase separation drives heterochromatin domain formation. Nature 547, 241-245. doi: 10.1038/nature22989

Su, X. A., Dion, V., Gasser, S. M., and Freudenreich, C. H. (2015). Regulation of recombination at yeast nuclear pores controls repair and triplet repeat stability. Genes Dev. 29, 1006-1017. doi: 10.1101/gad.25640 4.114
Swartz, R. K., Rodriguez, E. C., and King, M. C. (2014). A role for nuclear envelopebridging complexes in homology-directed repair. Mol. Biol. Cell 25, 2461-2471. doi: 10.1091/mbc.e13-10-0569

Therizols, P., Duong, T., Dujon, B., Zimmer, C., and Fabre, E. (2010). Chromosome arm length and nuclear constraints determine the dynamic relationship of yeast subtelomeres. Proc. Natl. Acad. Sci. U.S.A. 107, 2025-2030. doi: 10.1073/pnas. 0914187107

Therizols, P., Fairhead, C., Cabal, G. G., Genovesio, A., Olivo-Marin, J. C., Dujon, B., et al. (2006). Telomere tethering at the nuclear periphery is essential for efficient DNA double strand break repair in subtelomeric region. J. Cell Biol. 172, 189-199. doi: 10.1083/jcb.200505159

Torres-Rosell, J., Machin, F., Farmer, S., Jarmuz, A., Eydmann, T., Dalgaard, J. Z., et al. (2005). SMC5 and SMC6 genes are required for the segregation of repetitive chromosome regions. Nat. Cell Biol. 7, 412-419. doi: 10.1038/ ncb1239

Torres-Rosell, J., Sunjevaric, I., Piccoli, G. De, Sacher, M., Eckert-Boulet, N., Reid, R., et al. (2007). The Smc5-Smc6 complex and SUMO modification of Rad52 regulates recombinational repair at the ribosomal gene locus. Nat. Cell Biol. 923-931. doi: 10.1038/ncb1619

Tsouroula, K., Furst, A., Rogier, M., Heyer, V., Maglott-Roth, A., Ferrand, A., et al. (2016). Temporal and Spatial Uncoupling of DNA Double Strand Break Repair Pathways within Mammalian Heterochromatin. Mol. Cell 63, 293-305. doi: 10.1016/j.molcel.2016.06.002

van Sluis, M., and McStay, B. (2015). A localized nucleolar DNA damage response facilitates recruitment of the homology-directed repair machinery independent of cell cycle stage. Genes Dev. 29, 1151-1163. doi: 10.1101/gad.260703.115

Verdaasdonk, J. S., Vasquez, P. A., Barry, R. M., Barry, T., Goodwin, S., Forest, M. G., et al. (2013). Centromere tethering confines chromosome domains. Mol. Cell 52, 819-831. doi: 10.1016/j.molcel.2013.10.021

Waterman, D. P., Zhou, F., Li, K., Lee, C. S., Tsabar, M., Eapen, V. V., et al. (2019). Live cell monitoring of double strand breaks in S. cerevisiae. PLoS Genet. 15:e1008001. doi: 10.1371/journal.pgen.1008001

Weber, S. C., Spakowitz, A. J., and Theriot, J. A. (2010). Bacterial chromosomal loci move subdiffusively through a viscoelastic cytoplasm. Phys. Rev. Lett. 104:238102.

Whalen, J. M., Dhingra, N., Wei, L., Zhao, X., and Freudenreich, C. (2020). Relocation of Collapsed Forks to the Nuclear Pore Complex Depends on Sumoylation of DNA Repair Proteins and Permits Rad51 Association. Cell Rep. 31:107635. doi: $10.2139 /$ ssrn.3523272

Wong, H., Marie-Nelly, H., Herbert, S., Carrivain, P., Blanc, H., Koszul, R., et al. (2012). A predictive computational model of the dynamic 3D interphase yeast nucleus. Curr. Biol. 22, 1881-1890. doi: 10.1016/j.cub.2012. 07.069

Zada, D., Bronshtein, I., Lerer-Goldshtein, T., Garini, Y., and Appelbaum, L. (2019). Sleep increases chromosome dynamics to enable reduction of accumulating DNA damage in single neurons. Nat. Commun. 10:895.

Zhang, T., Zhang, Z., Shengzhao, G., Li, X., Liu, H., and Zhao, Y. (2019). Strand break-induced replication fork collapse leads to $\mathrm{C}$-circles, $\mathrm{C}$-overhangs and telomeric recombination. PLoS Genet. 15:e1007925. doi: 10.1371/journal.pgen. 1007925

Zimmer, C. (2018). "Chromatin mobility upon DNA damage: state of the art and remaining questions," in Current Genetics, Ed. M. Kupiec (Berlin: Springer), $0-0$.

Ziv, Y., Bielopolski, D., Galanty, Y., Lukas, C., Taya, Y., Schultz, D. C., et al. (2006). Chromatin relaxation in response to DNA double-strand breaks is modulated by a novel ATM- and KAP-1 dependent pathway. Nat. Cell Biol. 8, 870-876. doi: $10.1038 /$ ncb1446

Conflict of Interest: The authors declare that the research was conducted in the absence of any commercial or financial relationships that could be construed as a potential conflict of interest.

Copyright (c) 2020 Miné-Hattab and Chiolo. This is an open-access article distributed under the terms of the Creative Commons Attribution License (CC BY). The use, distribution or reproduction in other forums is permitted, provided the original author(s) and the copyright owner(s) are credited and that the original publication in this journal is cited, in accordance with accepted academic practice. No use, distribution or reproduction is permitted which does not comply with these terms. 